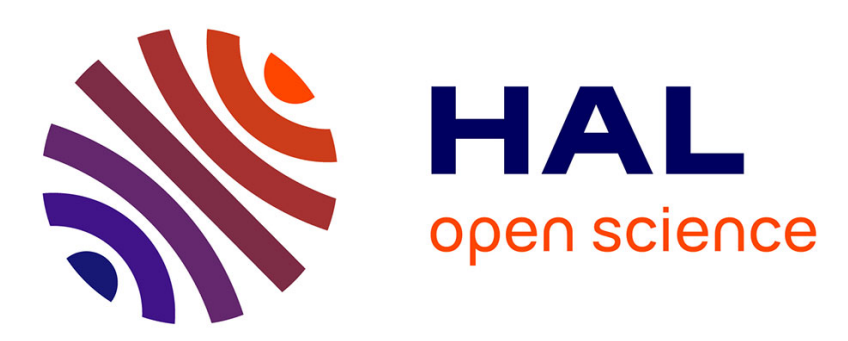

\title{
Distance estimates for state constrained trajectories of infinite dimensional differential inclusions
}

Hélène Frankowska, Elsa M Marchini, Marco Mazzola

\section{To cite this version:}

Hélène Frankowska, Elsa M Marchini, Marco Mazzola. Distance estimates for state constrained trajectories of infinite dimensional differential inclusions. ESAIM: Control, Optimisation and Calculus of Variations, 2018, 24 (3), pp.1207-1229. 10.1051/cocv/2017032 . hal-02126117

\section{HAL Id: hal-02126117 \\ https://hal.science/hal-02126117}

Submitted on 10 May 2019

HAL is a multi-disciplinary open access archive for the deposit and dissemination of scientific research documents, whether they are published or not. The documents may come from teaching and research institutions in France or abroad, or from public or private research centers.
L'archive ouverte pluridisciplinaire HAL, est destinée au dépôt et à la diffusion de documents scientifiques de niveau recherche, publiés ou non, émanant des établissements d'enseignement et de recherche français ou étrangers, des laboratoires publics ou privés. 


\title{
DISTANCE ESTIMATES FOR STATE CONSTRAINED TRAJECTORIES OF INFINITE DIMENSIONAL DIFFERENTIAL INCLUSIONS
}

\author{
H. FRANKOWSKA, E.M. MARCHINI, AND M. MAZZOLA
}

\begin{abstract}
This paper concerns estimates on the distance between a trajectory of a differential inclusion and the set of feasible trajectories of the same inclusion, feasible meaning confined to a given set of constraints. We apply these estimates to investigate Lipschitz continuity of the value functions arising in optimal control, and to variational inclusions, useful for proving non degenerate necessary optimality conditions. The main feature of our analysis is the infinite dimensional framework, which can be applied to models involving PDEs.
\end{abstract}

Keywords. Semilinear differential inclusion, state constraint, relaxation theorem, neighboring feasible trajectory theorem.

\section{INTRODUCTION}

Consider the differential inclusion

$$
\dot{x}(t) \in F(t, x(t)), \quad \text { a.e. } t \in\left[t_{0}, 1\right], \quad x\left(t_{0}\right)=x_{0}
$$

under the state constraint:

$$
x(t) \in K, \quad \text { for } t \in\left[t_{0}, 1\right],
$$

where $F: I \times \mathbb{R}^{n} \rightsquigarrow \mathbb{R}^{n}$ is a set-valued map, $I=[0,1], t_{0} \in I$ and $K$ is a closed subset of $\mathbb{R}^{n}$. Estimates on the distance between a trajectory of differential inclusion (1.1) and the set of all its feasible trajectories, that is satisfying in addition the state constraint (1.2), have been the object of an intense study, see $[4,5,9,18,20,21]$ and their bibliographies, to mention only a few. Such estimates are usually called neighbouring feasible trajectory theorems and their interest is due, on one hand, to constructive proofs, not requiring penalization and, on another hand, to the wide-spread applications in state constrained optimal control problems. Regularity properties of the value function, sensitivity relations, dynamic programming, non degenerate form of necessary optimality conditions, are some of the results obtained by using such distance estimates. Also the developed constructions of feasible trajectories did allow to obtain relaxation theorems under state constraints, i.e. to prove density of feasible trajectories in the set of relaxed feasible trajectories (that is when $F(t, x)$ are replaced by their convex envelopes).

Date: January 18, 2017.

2010 Mathematics Subject Classification. 34A60, 35Q93, 46N20, 47J22, 47N70, 93C23.

This work was co-funded by the DeMAGMA project of PROGRAMME CONVERGENCE, Sorbonne Universités, and by GNAMPA-INDAM research projects 2015. 
The infinite dimensional control systems and differential inclusions under state constraints, the natural framework for the description of many physical phenomena modeled by PDEs, as diffusion, vibration of strings or membranes, population dynamics, fluid dynamics, have been analyzed, by such direct approach, for the first time in [17], where, in particular, a relaxation theorem has been proved. The differential inclusion under investigation takes the following form

$$
\dot{x}(t) \in \mathbb{A} x(t)+F(t, x(t)), \quad \text { a.e. } t \in\left[t_{0}, 1\right], \quad x\left(t_{0}\right)=x_{0}
$$

subject to the similar, but infinite dimensional, state constraint:

$$
x(t) \in K, \quad \text { for all } t \in\left[t_{0}, 1\right] .
$$

Here, the densely defined unbounded linear operator $\mathbb{A}$ is the infinitesimal generator of a strongly continuous semigroup $S(t): X \rightarrow X, X$ is an infinite dimensional separable Banach space, $F: I \times X \rightsquigarrow X$ is a set-valued map with closed non-empty images and $K$ is a given subset of $X$. The trajectories of (1.3) are understood in the mild sense (see $[26])$.

The main result in [17] states that, under a convenient inward pointing condition, any feasible trajectory, that is any mild solution $x$ of (1.3) satisfying (1.4), can be approximated by a mild trajectory of (1.3) lying in the interior of $K$ for $t>t_{0}$. As in the finite dimensional case, this kind of approximations plays a key role in many proofs dealing with constrained problems, see e.g. the references mentioned before. The aim of our paper is to make a further step forward and to extend results on distance estimates to the infinite dimensional setting. In particular, we prove a neighboring feasible trajectory theorem stating that under an inward pointing condition, there exists $c_{0}>0$ such that for any trajectory $\hat{x}$ of (1.3) and for any $\varepsilon>0$, one can construct a feasible trajectory $x$ lying in the interior of $K$ and satisfying

$$
\|\hat{x}-x\|_{\mathcal{C}\left(\left[t_{0}, 1\right], X\right)} \leq c_{0}\left(\max _{t \in\left[t_{0}, 1\right]} \operatorname{dist}_{K}(\hat{x}(t))+\varepsilon\right),
$$

where $\max _{t \in\left[t_{0}, 1\right]} \operatorname{dist}_{K}(\hat{x}(t))$ is interpreted as a measure of the constraint violation by $\hat{x}$. As a consequence, we obtain two applications of these results to infinite dimensional control problems: sufficient conditions for local Lipschitz continuity of the value function associated to the Mayer optimal control problem under state constraints, and to variational inclusions, playing an important role in proving nondegenerate necessary conditions for optimality.

To obtain neighbouring feasible trajectory theorems in the finite dimensional framework under very general assumptions on $F$ and $K$, in $[18,19]$ a new inward pointing condition has been introduced. When $F$ is bounded and continuous near the boundary of $K$, it can be written in the following way:

$$
\begin{gathered}
\forall R>0, \exists \rho>0 \text { such that } \forall \bar{x} \in \partial K \cap R B, \\
\text { if } \sigma(\bar{x} ; v) \geq 0 \text { for some } t \in I, v \in F(t, \bar{x}), \text { then } \inf _{\bar{v} \in F(t, \bar{x})} \sigma(\bar{x} ; \bar{v}-v) \leq-\rho .
\end{gathered}
$$

In the above

$$
\sigma(x ; y)=\sup _{\xi \in \partial d_{K}(x)}\langle\xi, y\rangle, \quad \forall y \in X
$$


where $d_{K}(x)$ is the oriented distance from $x \in X$ to $K$ defined by

$$
d_{K}(x)= \begin{cases}\inf _{k \in K}\|x-k\|_{X} & \text { if } x \notin K \\ -\inf _{k \in(X \backslash K)}\|x-k\|_{X} & \text { otherwise }\end{cases}
$$

and $\partial d_{K}(x)$ denotes the Clarke generalized gradient of $d_{K}$ at the point $x \in X$.

Below we will use also the classical notion of distance from $x \in X$ to $K$, defined by

$$
\operatorname{dist}_{K}(x)=\inf _{k \in K}\|x-k\|_{X} .
$$

Condition (1.5) can be interpreted in the following way: for every $(t, \bar{x}) \in I \times \partial K$, any admissible velocity $v \in F(t, \bar{x})$ that is "pushing outward" in the sense that $\sigma(\bar{x} ; v) \geq 0$, can be corrected by a "compensating velocity" $\bar{v} \in F(t, \bar{x})$, satisfying $\sigma(\bar{x} ; \bar{v}-v) \leq-\rho$.

This kind of condition allows to treat constraints with nonsmooth boundary, arising naturally in applied sciences. Nevertheless, when $K$ has a smooth boundary, then (1.5) reduces to the classical inward pointing condition

$$
\forall R>0, \exists \rho>0 \text { such that } \inf _{\bar{v} \in F(t, \bar{x})}\left\langle\nabla d_{K}(\bar{x}), \bar{v}\right\rangle \leq-\rho, \forall(t, \bar{x}) \in I \times(\partial K \cap R B)
$$

that is equivalent to the Soner condition [27] when $K$ and $F$ are bounded, see [19] for more details.

In Proposition 5.4 we discuss an analogous reduction of the inward pointing condition (1.5) when $X$ is a reflexive Banach space.

We aim to work in great generality, to cover a large class of models: the infinite dimensional state space $X$ is Banach, and the unbounded operator $\mathbb{A}$ generates a semigroup $S(t)$ which is merely strongly continuous. For this reason, in our paper the semigroup $S(t)$ is involved in the formulation of the inward pointing conditions.

To simplify the notation, let us define for any positive $\eta$ the set

$$
\partial^{\eta} K=\{x \in K+\eta B: S(\tau) x \in \partial K+\eta B \text { for some } \tau \in[0, \eta]\},
$$

and for every $\eta>0$ and $x \in X$, the set

$$
\mathcal{A}^{\eta}(x)=\{(\tau, z) \in[0, \eta] \times X: S(\tau) x \in \partial K+\eta B, z \in B(S(\tau) x, \eta)\},
$$

and the function $\Sigma_{\eta}(x ; \cdot): X \rightarrow[-\infty,+\infty)$ that associates to any $v \in X$ the value

$$
\Sigma_{\eta}(x ; v)=\sup _{(\tau, z) \in \mathcal{A}^{\eta}(x)} \sigma(z ; S(\tau) v)
$$

with the convention that the supremum over the empty set is equal to $-\infty$. The introduction of the sets $\partial^{\eta} K$ and $\mathcal{A}^{\eta}(x)$ is due to the fact that, because of the presence of the semigroup $S(\cdot)$ and since, in general, the boundary of $K$ is not compact, we cannot consider anymore only the points on the boundary of $K$, but we are led to extend the inward pointing condition to points that are "sent" by the semigroup into a neighborhood of $\partial K$. Let us underline that $\partial K+\eta B \subset \partial^{\eta} K$ for any $\eta>0$ and that this inclusion may be strict. The function $\Sigma_{\eta}(x ; \cdot)$ allows to define "outward" and "compensating" velocities. Observe that it is equal to $-\infty$ whenever $S(\tau) x$ does not belong to $\partial K+\eta B$ for all $\tau \in[0, \eta]$. 
Thus, in our very general context, the inward pointing condition takes the form

$$
\forall R>0, \exists \eta, \rho, M>0, \exists J \subset I \text { such that } \mu(J)=1 \text { and if } \Sigma_{\eta}(x ; v) \geq 0
$$

for some $t \in J, x \in R B \cap \partial^{\eta} K, v \in F(t, x)$, then $\exists \bar{v} \in F(t, x) \cap B(v, M)$ satisfying

$$
\max \left\{\Sigma_{\eta}(x ; \bar{v}-v) ; \Sigma_{\eta}(x ; \bar{v})\right\} \leq-\rho .
$$

Because of the generality of setting, this inward pointing condition may seem complicated. Nevertheless, some simplifications of (1.7) hold: we will prove in Lemma 5.1 that, when working with locally bounded and convex valued $F$, condition (1.7) is reduced to

$$
\begin{gathered}
\forall R>0, \exists \eta, \rho>0, \exists J \subset I \text { such that } \mu(J)=1 \text { and whenever } \Sigma_{\eta}(x ; v) \geq 0 \\
\text { for some } t \in J, x \in R B \cap \partial^{\eta} K, v \in F(t, x) \text {, then } \exists \bar{v} \in F(t, x) \text { satisfying } \\
\qquad \Sigma_{\eta}(x ; \bar{v}-v) \leq-\rho .
\end{gathered}
$$

Further, if some compactness is present, conditions (1.7), (1.8) can be drastically simplified and, in some cases, reduced to its finite dimensional counterpart (1.5) or (1.6), as we show in Section 5.

The paper is structured as follows: Section 2 contains notations, definitions and assumptions. In Section 3 we provide examples of physical models satisfying the main hypotheses in use. The new results are stated in Section 4, while in Section 5 we provide the conditions allowing to simplify (1.7) into (1.5) or (1.6), and applications to the concrete examples. Sections 6 and 7 deal with Lipschitz continuity of the value function and with variational inclusions, and the final Section 8 is devoted to the proofs of the main results.

\section{Preliminaries}

In this section we list the notation and the main assumptions in use throughout the paper.

\subsection{Notation.}

- $X$ is a separable Banach space;

- $B(x, r)$ denotes the closed ball of center $x \in X$ and radius $r>0 ; B$ is the closed unit ball in $X$ centered at 0 ;

- given a Banach space $Y, \mathbb{L}(X, Y)$ denotes the Banach space of bounded linear operators from $X$ into $Y, \mathcal{C}(I, X)$ the space of continuous functions from $I$ to $X$, $L^{p}(I, X)$ the space of Bochner $L^{p}$ integrable functions from $I$ to $X$, and $L^{\infty}(I, X)$ the space of measurable essentially bounded functions from $I$ to $X$;

- $\langle\cdot, \cdot\rangle$ stands for the duality pairing on $X^{*} \times X$;

- $\mu$ is the Lebesgue measure on the real line;

- given a set-valued map $\mathcal{F}: X \rightsquigarrow X, x \in X$ and $y \in \mathcal{F}(x)$, the derivative $d \mathcal{F}(x, y)$ : $X \rightsquigarrow X$ is defined by

$$
v \in d \mathcal{F}(x, y) w \quad \Leftrightarrow \quad \lim _{h \rightarrow 0+} \operatorname{dist}\left(v, \frac{\mathcal{F}(x+h w)-y}{h}\right)=0
$$


- the intermediate (or adjacent) cone to $K$ at $x$ is defined as

$$
\begin{aligned}
I_{K}(x) & =\left\{v \in X: \lim _{h \rightarrow 0^{+}} \operatorname{dist}\left(v, \frac{K-x}{h}\right)=0\right\} \\
& =\left\{v \in X: \forall h_{i} \rightarrow 0^{+}, \exists w_{i} \rightarrow v \text { such that } x+h_{i} w_{i} \in K\right\} .
\end{aligned}
$$

We will use the following notion of solution.

Definition 2.1. Let $t_{0} \in I$ and $x_{0} \in X$. A function $x \in \mathcal{C}\left(\left[t_{0}, 1\right], X\right)$ is a (mild) solution of (1.3) with initial datum $x\left(t_{0}\right)=x_{0}$ if there exists a function $f^{x} \in L^{1}\left(\left[t_{0}, 1\right], X\right)$ such that

$$
f^{x}(t) \in F(t, x(t)), \quad \text { for a.e. } t \in\left(t_{0}, 1\right)
$$

and

$$
x(t)=S\left(t-t_{0}\right) x_{0}+\int_{t_{0}}^{t} S(t-s) f^{x}(s) \mathrm{d} s, \quad \text { for any } t \in\left[t_{0}, 1\right],
$$

i.e. $f^{x}$ is an integrable selection of the set valued map $t \rightsquigarrow F(t, x(t))$ and $x$ is a mild solution (see [26]) of the initial value problem

$$
\left\{\begin{array}{l}
\dot{x}(t)=\mathbb{A} x(t)+f^{x}(t), \quad \text { for a.e. } t \in\left[t_{0}, 1\right] \\
x\left(t_{0}\right)=x_{0}
\end{array}\right.
$$

We denote by $\mathcal{S}_{\left[t_{0}, 1\right]}^{K}\left(x_{0}\right)$ the set of feasible trajectories of (1.3) with initial datum $x_{0}$. Since $S(t)$ is a strongly continuous semigroup, there exists $M_{S} \geq 1$ such that

$$
\|S(t)\|_{\mathbb{L}(X, X)} \leq M_{S}, \quad \text { for any } t \in I .
$$

The differential inclusion (1.3) is a convenient tool to investigate for example the semilinear control system

$$
\left\{\begin{array}{l}
\dot{x}(t)=\mathbb{A} x(t)+f(t, x(t), u(t)), u(t) \in U, \quad \text { a.e. } t \in\left[t_{0}, 1\right] \\
x\left(t_{0}\right)=x_{0}
\end{array}\right.
$$

where $U$ is a complete separable metric space. Setting $F(t, x)=f(t, x, U)$, we can reduce (2.4) to (1.3) by applying a measurable selection theorem.

2.2. Assumptions. The following conditions are assumed in the main results:

- positive invariance of $K$ by the semigroup:

$$
S(t) K \subset K, \quad \forall t \in I
$$

- for every $(t, x) \in I \times X, F(t, x)$ is closed, non empty, and, for any $x \in X$, the set-valued map $F(\cdot, x)$ is Lebesgue measurable;

- for every $t \in I$, the set-valued map $F(t, \cdot)$ is locally Lipschitz in the following sense: for any $R>0$, there exists $k_{R} \in L^{1}\left(I, \mathbb{R}^{+}\right)$such that, for a.e. $t \in I$ and any $x, y \in R B$,

$$
F(t, x) \subset F(t, y)+k_{R}(t)\|x-y\|_{X} B
$$


- there exists $\phi \in L^{1}\left(I, \mathbb{R}^{+}\right)$such that, for a.e. $t \in I$ and any $x \in X$,

$$
F(t, x) \subset \phi(t)\left(1+\|x\|_{X}\right) B .
$$

\section{EXAMPLES OF INVARIANT SETS}

The great generality of the main assumptions allows to apply our results to a number of phenomena modeled by PDEs. The only restrictive condition regards the positive invariance of the state constraint $K$ by the linear semigroup $S(t)$, namely assumption (2.5). A large literature has been devoted to ensure such a property. For instance, when $K$ is closed and convex, necessary and sufficient conditions for (2.5) are well known. To mention only few contributions in this direction, see e.g. [7, 11, 16, 25] and the references therein. In particular, in the case when $K$ is a cone inducing a partial ordering in the Banach space $X$, invariance properties have been deeply studied due to their importance in several applications. For instance in models describing populations phenomena this kind of constraint is a natural requirement. When $\mathbb{A}$ is the realization of a second order elliptic operator, invariance of pointwise constraints (such as positivity of solutions) is provided by the maximum principle (and its generalizations). Among this variety of models, we have selected a family of problems satisfying (2.5), to which we can apply our main theorems, see Section 5 for further details.

3.1. The case of the contraction semigroups. A meaningful class of examples is provided by systems of type (1.3), where the operator $\mathbb{A}$ generates a $C_{0}$ semigroup $S(t)$ of contraction. Here the constraint $K=R B$ fulfills the required invariance (2.5), allowing to impose an upper bound on the associated energy. In this framework, many systems arising from PDEs can be found in the literature.

Example 3.1 (Boltzmann viscoelasicity). We analyze at first a model for the isothermal viscoelasticity, see [17] and the references therein, where the same problem has been studied. This phenomenon is described by an integrodifferential equation. Namely, taking $\Omega \subset \mathbb{R}^{3}$, a bounded domain with smooth boundary $\partial \Omega$ representing the region occupied by an elastic body, and a memory kernel $\mu$, accounting for the hereditary properties of the viscoelastic material, the displacement function $u=u(t, \mathbf{x}), t \in(0,1)$ and $\mathbf{x} \in \Omega$, satisfies

$$
\partial_{t t} u(t)-\Delta\left[u(t)-\int_{0}^{\infty} \mu(s) u(t-s) \mathrm{d} s\right] \in F(t, u(t)),
$$

(we omit the variable $\mathbf{x}$ ). Imposing Dirichlet boundary conditions, complies with the assumption that the body is kept fixed at the boundary of $\Omega$. As detailed in [10, 17], by introducing an auxiliary variable in accord to the classical Dafermos' history approach [14], (3.1) can be rewritten as a differential inclusion of type (1.3), where, in a suitable functional Hilbert space $X$, a linear operator $\mathbb{A}$ generates a strongly continuous semigroup of contractions $S(t): X \rightarrow X$.

Example 3.2 (The acoustic wave equation). The second example arises in theoretical acoustics, when modeling transverse vibrations in a membrane whose boundary can vibrate. The differential inclusion ruled by the classical wave equation

$$
\partial_{t t} u \in \Delta u+F(t, u),
$$


for $u=u(t, \mathbf{x}), t \geq 0, \mathbf{x} \in \Omega$, where a smooth bounded domain $\Omega$ of $\mathbb{R}^{N}$, is endowed with the so called acoustic boundary conditions,

$$
m \partial_{t t} \delta+d \partial_{t} \delta+k \delta+\rho u_{t}=0
$$

underlying the assumption that any $\mathbf{x} \in \partial \Omega$ reacts to the excess pressure of the wave as a resistive harmonic oscillator. Here $\delta=\delta(t, \mathbf{x}), t \geq 0, \mathbf{x} \in \partial \Omega$ is the normal displacement of the boundary, $\rho$ is the density, $m, d, k \in \mathcal{C}(\partial \Omega)$ represent respectively the mass, the friction coefficient, and the spring constant. Assuming the boundary impenetrable, the following compatibility condition follows: $\partial_{t} \delta=\partial_{\nu} u$, where $\partial_{\nu} u$ is the directional derivative of $u$ in the direction of the outward normal to $\Omega$. These boundary conditions have been introduced by Moser and Ingard in [24], and formalized in a rigorous mathematical framework in [3], see also [13, 22]. As shown in [22], equation (3.2) can be rewritten in the form (1.3), with an operator $\mathbb{A}$ that is a $4 \times 4$ matrix generating a strongly continuous semigroup of contractions in the Hilbert space

$$
X=H^{1}(\Omega) \times L^{2}(\Omega) \times L^{2}(\partial \Omega) \times L^{2}(\partial \Omega)
$$

with norm

$$
\|x\|_{X}^{2}=\int_{\Omega}\left(\rho|\nabla u|^{2}+\frac{\rho}{c^{2}}\left|\partial_{t} u\right|^{2}\right) d \mathbf{x}+\int_{\partial \Omega}\left(k|\delta|^{2}+m\left|\partial_{t} \delta\right|^{2}\right) d S,
$$

for $x=\left(u, \partial_{t} u, \delta, \partial_{t} \delta\right)$.

3.2. Conservative systems. We consider here the non convex set $K=R B \backslash r B$, with $0<r<R$, complying with the request that the energy associated to the system should be constrained between two bounds. As example, we can take the acoustic wave equation (3.2)-(3.3). Defining as energy of the solution $E=\|x\|_{X}^{2}$, a simple estimate, see [22], shows that

$$
\frac{d E}{d t}=-2 \int_{\partial \Omega} d\left|\partial_{t} \delta\right|^{2} d S
$$

implying that (3.2)-(3.3) is a conservative system once the friction coefficient $d \equiv 0$. Hence the annulus $R B \backslash r B$, with $0<r<R$ is positively invariant under the action of the associated semigroup, and (2.5) is satisfied for this type of constraint.

3.3. Pointwise constraints. A class of parabolic problems arising in heat diffusion is considered, where we impose a pointwise state constraint, such as the positivity of the solutions. With the exception of a one-dimensional problem, whose framework is the Hilbert space $H^{1}(0,1)$ (embedded in $\mathcal{C}([0,1])$ ), in higher dimension, in order to have Int $K \neq \emptyset$, we work in the Banach space of the continuous functions. At first the zeroobstacle problem is investigated, namely, we require the positivity of the solutions.

Example 3.3 ( $A$ one-dimensional heat equation). To model the heat flux in a cylindrical bar, with perfectly insulated lateral surface and whose length is much larger than its crosssection, a one-dimensional equation is introduced. For $u=u(t, s), t, s \in[0,1] \times[0,1]$, let

$$
\partial_{t} u \in \partial_{s s} u-u+F(t, u),
$$

endowed with the Neumann boundary conditions, complying with the assumption that the heat flux at the two ends of the bar is zero. In order to rewrite (3.4) as (1.3), we introduce 
the operator $\mathbb{A}=\partial_{s s}-I$ with domain $D(\mathbb{A})=\left\{x \in H^{2}(0,1): x^{\prime}(0)=x^{\prime}(1)=0\right\}$. $\mathbb{A}$ is the infinitesimal generator of a strongly continuous semigroup $\tilde{S}(t)$ on $Y=L^{2}(0,1)$. The space $X=H^{1}(0,1)$ is an invariant subspace of $Y$, and the restriction of $\tilde{S}(t)$ to $X$ (called $S(t))$ is a strongly continuous semigroup in $X$, see [28]. Then, we can apply our results to $S(t): X \rightarrow X$ where the cone of nonnegative functions

$$
K=\{x \in X: x(s) \geq 0, \text { for } s \in[0,1]\},
$$

has non empty interior. Further, $K$ is positively invariant under the action of $S(t)$. An interesting property of this constraint set, which will allow to simplify drastically our inward point condition, is that the set of the external normals to $K$ is pre-compact, see [17] for the proof.

Example 3.4 (Heat equation with dynamical boundary conditions). In this example, we study a heat conduction process with dynamic (or Wentzell) boundary conditions

$$
\begin{cases}\partial_{t} u \in \Delta u+F(t, u) & \text { in }(0,1) \times \Omega \\ \partial_{t} u=-\partial_{\nu} u-\gamma u & \text { on }(0,1) \times \partial \Omega .\end{cases}
$$

Here $u=u(t, \mathbf{x}), \Omega$ is a bounded domain of $\mathbb{R}^{N}$ with smooth boundary and $\gamma \in \mathcal{C}(\partial \Omega)$. This kind of systems has been the object of an intense research, due to its relevance from a modeling point of view, see e.g. [23]. As outlined in $[2,29]$, problem (3.5) can be seen as a heat equation with Wentzell boundary condition, and studied in the space $\mathcal{C}(\bar{\Omega})$, a natural space for such boundary conditions. We associate to (3.5) the operator

$$
\mathbb{A}=\Delta, \quad \operatorname{dom}(\mathbb{A})=\left\{u \in \mathcal{C}_{\nu}^{1}(\bar{\Omega}): \Delta u \in \mathcal{C}(\bar{\Omega}) ; \Delta u+\partial_{\nu} u+\gamma u=0 \text { on } \partial \Omega\right\},
$$

with $\mathcal{C}_{\nu}^{1}(\bar{\Omega})$ being the space of functions $u \in \mathcal{C}(\bar{\Omega})$ for which the outer normal derivative $\partial_{\nu} u$ exists and is continuous on $\partial \Omega$, see $[1,15]$. As proved e.g. in [15], this operator generates a positive compact $C_{0}$ semigroup in the space of continuous functions $\mathcal{C}(\bar{\Omega})$. Hence, the constraint set

$$
K=\{u \in \mathcal{C}(\bar{\Omega}): u(\mathbf{x}) \geq 0, \text { for } \mathbf{x} \in \bar{\Omega}\}
$$

has non-empty interior and (2.5) is satisfied.

Example 3.5 (A further diffusion equation). In this last example, we consider again a pointwise constraint: we want to control a temperature in a room by imposing that it is constrained between two fixed values. The model describing the heat process is governed by the heat equation with Neumann boundary conditions

$$
\begin{cases}\partial_{t} u \in \Delta u+F(t, u) & \text { in }(0,1) \times \Omega \\ \partial_{\nu} u=0 & \text { on }(0,1) \times \partial \Omega\end{cases}
$$

where $u=u(t, \mathbf{x})$ and $\Omega$ is a bounded domain of $\mathbb{R}^{N}$ with smooth boundary. The operator associated to the system (3.6) generates a positive $C_{0}$-semigroup on $Y=L^{2}(\Omega)$. As done in example 3.3, we can consider its restriction to the invariant subspace $X=\mathcal{C}(\bar{\Omega})$, see e.g. in [1], where it has been proved that the strongly continuous semigroup induced on $X$ is positive and compact. Further, applying the parabolic maximum principle, see [6], we deduce that the state constraint

$$
K=\{u \in \mathcal{C}(\bar{\Omega}):-1 \leq u(\mathbf{x}) \leq 1, \mathbf{x} \in \bar{\Omega}\}
$$


is positively invariant, yielding the validity of (2.5).

\section{The MAIN RESUltS}

This section contains the main results of the paper. Their proofs are postponed to Section 8 .

Consider the semilinear differential inclusion

$$
\dot{x}(t) \in \mathbb{A} x(t)+F(t, x(t)), \quad \text { a.e. } t \in\left[t_{0}, 1\right],
$$

subject to the state constraint:

$$
x(t) \in K, \quad \text { for all } t \in\left[t_{0}, 1\right],
$$

where $K$ is a closed subset of $X$.

4.1. Neighbouring feasible trajectory theorems. Our first neighboring feasible trajectory theorem is stated in the greatest generality.

Theorem 4.1. Assume (2.5)-(2.8) and (1.7). Then, for any $R_{0}>0$ there exists $c_{0}>0$ such that, for any $t_{0} \in I$, any $\varepsilon>0$, and any trajectory $\hat{x}$ of (4.1) with $\hat{x}\left(t_{0}\right) \in K \cap R_{0} B$, there exists $x \in \mathcal{S}_{\left[t_{0}, 1\right]}^{K}\left(\hat{x}\left(t_{0}\right)\right)$ satisfying

$$
x(t) \in \operatorname{Int} K, \quad \text { for any } t \in\left(t_{0}, 1\right]
$$

and

$$
\|\hat{x}-x\|_{\mathcal{C}\left(\left[t_{0}, 1\right], X\right)} \leq c_{0}\left(\max _{t \in\left[t_{0}, 1\right]} \operatorname{dist}_{K}(\hat{x}(t))+\varepsilon\right) .
$$

The same conclusions are valid if (2.5)-(2.8) hold true with $\phi \in L^{\infty}\left(I, \mathbb{R}^{+}\right), F$ is convex valued, and (1.7) is replaced by (1.8).

Some applications (see Section 7) require weaker estimates than those expressed in Theorem 4.1 and hold true under weaker versions of conditions (1.7) and (1.8):

$$
\begin{aligned}
& \forall D \subset X \text { compact, } \exists \eta, \rho, M>0, \exists J \subset I \text { such that } \mu(J)=1 \text { and if } \\
& \sup _{\tau \in[0, \eta], z \in B(x, \eta)} \sigma(z ; S(\tau) v) \geq 0 \text { for some } t \in J, x \in D \cap(\partial K+\eta B), v \in F(t, x) \text {, then } \\
& \exists \bar{v} \in F(t, x) \cap B(v, M) \text { with } \sup _{\tau \in[0, \eta], z \in B(x, \eta)}\{\sigma(z ; S(\tau)(\bar{v}-v)) ; \sigma(z ; S(\tau) \bar{v})\} \leq-\rho,
\end{aligned}
$$

respectively

$\forall D \subset X$ compact, $\exists \eta, \rho>0, \exists J \subset I$ such that $\mu(J)=1$ and if $\sup _{\tau \in[0, \eta], z \in B(x, \eta)} \sigma(z ; S(\tau) v) \geq 0$ for some $t \in J, x \in D \cap(\partial K+\eta B), v \in F(t, x)$, then $\exists \bar{v} \in F(t, x)$ satisfying $\sup _{\tau \in[0, \eta], z \in B(x, \eta)} \sigma(z ; S(\tau)(\bar{v}-v)) \leq-\rho$. 
Theorem 4.2. Assume (2.5)-(2.8), (4.5) and that

for any compact $D_{0} \subset X$, there exists a compact $D \subset X$ such that $\forall t_{0} \in I, y\left(\left[t_{0}, 1\right]\right) \subset D$, for any trajectory $y$ of (4.1) satisfying $y\left(t_{0}\right) \in D_{0}$.

Then, for every compact set $D_{0} \subset X$, we can find $c_{0}=c\left(D_{0}\right)>0$ such that, for any $t_{0} \in I$, any $\varepsilon>0$, and any trajectory $\hat{x}$ of (4.1) with $\hat{x}\left(t_{0}\right) \in K \cap D_{0}$, there exists $x \in \mathcal{S}_{\left[t_{0}, 1\right]}^{K}\left(\hat{x}\left(t_{0}\right)\right)$ satisfying (4.3) and (4.4).

The same conclusions hold true if (4.5) is replaced by (4.6), $\phi \in L^{\infty}\left(I, \mathbb{R}^{+}\right)$and $F$ is convex valued.

The following proposition provides two sufficient conditions for (4.7) to be satisfied. The first of them holds true for instance in the model of Examples 3.4, 3.5.

Proposition 4.3. Assume (2.8) and that either

$$
S(\cdot) \text { is a compact semigroup }
$$

or

$\forall R>0$ there exists a compact $\mathcal{K}_{R} \subset X$ such that $F(t, x) \subset \mathcal{K}_{R}, \forall(t, x) \in I \times R B$.

Then condition (4.7) is satisfied.

Proof. Let $D_{0}$ be a compact subset of $X$. Assume first (4.9) and consider a trajectory $y$ of (4.1) with initial datum $y_{0}=y\left(t_{0}\right) \in D_{0}$. By the representation formula (2.2),

$$
y(t)=S\left(t-t_{0}\right) y_{0}+\int_{t_{0}}^{t} S(t-s) f^{y}(s) d s,
$$

with $f^{y}$ as in (2.1) (and $x$ replaced by $y$ ). By (2.8) and the Gronwall lemma there exists $R>0$ only depending on $D_{0}$ and $\phi$, such that $y(t) \in R B$, for any $t \in\left[t_{0}, 1\right]$. By the continuity properties of the semigroup $S(t)$ and by the compactness assumption (4.9), there exists a compact set $\tilde{D} \subset X$, depending on $D_{0}$ and $R$ satisfying

$$
S\left(t-t_{0}\right) y_{0} \in \tilde{D} \text { and } S(t-s) f^{y}(s) \in \tilde{D}, \quad \text { for any } t \text { and a.e. } s \text { in }\left[t_{0}, 1\right]
$$

implying the claimed

$$
y\left(\left[t_{0}, 1\right]\right) \subset D=2 \overline{c o} \tilde{D} .
$$

In case assumption (4.8) holds, our proof is based on [8, Lemma 5.4]. Consider the relaxed system

$$
\dot{x}(t) \in \mathbb{A} x(t)+\overline{c o} F(t, x(t)), \quad \text { a.e. } t \in\left[t_{0}, 1\right] .
$$

Adapting to the differential inclusions setting the assumptions of Lemma 5.4 in [8], we deduce that the set

$$
\mathcal{S}\left(D_{0}\right)=\left\{\tilde{x} \text { solution to }(4.10) \text { with } \tilde{x}\left(t_{0}\right) \in D_{0}\right\}
$$

is compact in $\mathcal{C}\left(\left[t_{0}, 1\right], X\right)$. This implies that the set

$$
D=\left\{\tilde{x}(t): \tilde{x} \in \mathcal{S}\left(D_{0}\right), t \in\left[t_{0}, 1\right]\right\}
$$


is compact. Indeed, let $x_{i}\left(t_{i}\right) \in D$, taking a subsequence and keeping the same notation we have that $t_{i} \rightarrow \tilde{t}$, for some $\tilde{t} \in\left[t_{0}, 1\right]$ and, for some trajectory $\tilde{x} \in \mathcal{S}\left(D_{0}\right)$,

$$
\sup _{t \in\left[t_{0}, 1\right]}\left\|\tilde{x}_{i}(t)-\tilde{x}(t)\right\|_{X} \rightarrow 0
$$

This yields

$$
\begin{aligned}
\left\|x_{i}\left(t_{i}\right)-\tilde{x}(\tilde{t})\right\|_{X} & \leq\left\|x_{i}\left(t_{i}\right)-\tilde{x}\left(t_{i}\right)\right\|_{X}+\left\|\tilde{x}\left(t_{i}\right)-\tilde{x}(\tilde{t})\right\|_{X} \\
& \leq \sup _{t \in\left[t_{0}, 1\right]}\left\|x_{i}(t)-\tilde{x}(t)\right\|_{X}+\left\|\tilde{x}\left(t_{i}\right)-\tilde{x}(\tilde{t})\right\|_{X} \rightarrow 0,
\end{aligned}
$$

proving the compactness. Finally, since any solution of (1.3) is also a solution of (4.10), $D$ is as claimed.

4.2. Approximation and relaxation theorems. Results obtained in [17] can be generalized by using a less restrictive inward pointing condition stated in terms of the mapping $\Sigma_{\eta}(x ; \cdot)$. We do not provide here the proofs, since they follow easily by slight modifications of the proofs contained in [17].

Theorem 4.4. Assume (2.5)-(2.8) and that

$$
\forall \bar{x} \in \partial K, \exists \eta, \rho, M>0, \exists J \subset I \text { such that } \mu(J)=1 \text { and if }
$$

$$
\begin{gathered}
\sup _{\tau \in[0, \eta], z \in B(x, \eta)} \sigma(z ; S(\tau) v) \geq 0 \text { for some } t \in J, x \in K \cap B(\bar{x}, \eta), v \in F(t, x), \text { then } \\
\exists \bar{v} \in F(t, x) \cap B(v, M) \text { satisfying } \Sigma_{\eta}(x ; \bar{v}-v) \leq-\rho .
\end{gathered}
$$

Then, for any $\varepsilon>0$ and any trajectory $\hat{x}$ of (4.1), (4.2), there exists $x \in \mathcal{S}_{\left[t_{0}, 1\right]}^{K}\left(\hat{x}\left(t_{0}\right)\right)$ satisfying (4.3) and

$$
\|\hat{x}-x\|_{\mathcal{C}\left(\left[t_{0}, 1\right], X\right)} \leq \varepsilon .
$$

A consequence of the previous result is the following relaxation theorem:

Theorem 4.5. Assume (2.5)-(2.8) and that

$$
\begin{gathered}
\forall \bar{x} \in \partial K, \exists \eta, \rho, M>0, \exists J \subset I \text { such that } \mu(J)=1 \text { and if } \\
\sup _{\tau \in[0, \eta], z \in B(x, \eta)} \sigma(z ; S(\tau) v) \geq 0 \text { for some } t \in J, x \in K \cap B(\bar{x}, \eta), v \in \overline{c o} F(t, x), \text { then } \\
\exists \bar{v} \in \overline{c o} F(t, x) \cap B(v, M) \text { satisfying } \Sigma_{\eta}(x ; \bar{v}-v) \leq-\rho .
\end{gathered}
$$

Then, for any $\varepsilon>0$ and any trajectory $\hat{x}$ of

$$
\dot{x}(t) \in \mathbb{A} x(t)+\overline{c o} F(t, x(t)), \quad \text { a.e. } t \in\left[t_{0}, 1\right]
$$

and (4.2), there exists $x \in \mathcal{S}_{\left[t_{0}, 1\right]}^{K}\left(\hat{x}\left(t_{0}\right)\right)$ satisfying (4.3) and (4.12). 
4.3. The case of Hilbert spaces. When $X$ is a Hilbert space, the inward pointing conditions can be reformulated by using normal vectors to the boundary of $K$, instead of generalized gradients of the oriented distance function. This formulation is useful in the applications, as is Examples 3.1, 3.2, 3.3.

Let $\langle\cdot, \cdot\rangle_{X}$ denote the scalar product in $X$ and let $K$ be a proper closed subset of $X$ such that $K=\overline{\operatorname{Int} K}$. Denote by $Z$ the set of points $z \in X \backslash \partial K$ admitting a unique projection $P_{\partial K}(z)$ on $\partial K$. This set is dense in $X$. For every $z \in Z$, set

$$
n_{z}=\frac{z-P_{\partial K}(z)}{\left\|z-P_{\partial K}(z)\right\|_{X}} \operatorname{sgn}\left(d_{K}(z)\right) \text {. }
$$

Let us define for any $\eta>0$ and $x \in X$, the set

$$
\mathcal{B}^{\eta}(x)=\{(\tau, z) \in[0, \eta] \times Z: S(\tau) x \in \partial K+\eta B, z \in B(S(\tau) x, \eta)\} \subset \mathcal{A}^{\eta}(x),
$$

and the extended real valued function $\Pi_{\eta}(x ; \cdot): X \rightarrow[-\infty,+\infty)$ that associates to any $v \in X$ the value

$$
\Pi_{\eta}(x ; v)=\sup _{(\tau, z) \in \mathcal{B}^{\eta}(x)}\left\langle n_{z}, S(\tau) v\right\rangle,
$$

again with the convention that the supremum over the empty set takes the value $-\infty$. Then an inward pointing condition can be expressed in this setting by replacing (1.7) by (4.13) $\forall R>0, \exists \eta, \rho, M>0, \exists J \subset I$ such that $\mu(J)=1$ and whenever $\Pi_{\eta}(x ; v) \geq 0$

for some $t \in J, x \in R B \cap \partial^{\eta} K, v \in F(t, x)$, then $\exists \bar{v} \in F(t, x) \cap B(v, M)$ satisfying

$$
\max \left\{\Pi_{\eta}(x ; \bar{v}-v) ; \Pi_{\eta}(x ; \bar{v})\right\} \leq-\rho
$$

to get the same conclusions as those in Theorems 4.1, 4.2, 4.4, 4.5. To prove it, it is sufficient to follow arguments from [17] using the new conditions.

\section{REMARKS ON THE INWARD POINTING CONDITIONS}

In this section we analyze the inward pointing conditions. In the presence of compactness assumptions some simplifications hold. The first result below deals with (1.8) and (4.6), useful when working with relaxed differential inclusions, hence important in many applications. In the other three propositions of this section, we provide conditions which guarantee the validity of the neighboring feasible trajectory theorem under the classical inward pointing conditions (1.5) and (1.6).

Lemma 5.1. Let $F: I \times X \rightsquigarrow X$ be a set-valued map with convex nonempty images, satisfying (2.8) with $\phi \in L^{\infty}\left(I, \mathbb{R}^{+}\right)$. Then condition (1.8) implies (1.7) and (4.6) implies (4.5).

Proof. We only prove that (1.8) implies (1.7), the proof of the second implication being analogous.

Assume (1.8), fix $R>0$, and let $\eta, \rho$ and $J$ be as in (1.8). Set $\alpha=\|\phi\|_{L^{\infty}\left(I ; \mathbb{R}^{+}\right)}$, $M=2 \alpha(1+R)$ and $\tilde{J} \subset J$ such that $\mu(\tilde{J})=1$, and

$$
F(t, x) \subset \alpha\left(1+\|x\|_{X}\right) B \quad \forall(t, x) \in \tilde{J} \times X .
$$

Let $t \in \tilde{J}, x \in R B \cap \partial^{\eta} K$ and $v \in F(t, x)$ satisfy

$$
\Sigma_{\eta}(x ; v) \geq 0 .
$$


Then

$$
\mathcal{D}=\left\{\bar{v} \in F(t, x): \Sigma_{\eta}(x ; \bar{v}-v) \leq-\rho\right\}
$$

is nonempty. It is clear that $\mathcal{D} \subset B(v, M)$. The proof is performed in two steps.

Step 1. We claim that, there exists $\bar{v} \in \mathcal{D}$ such that

$$
\Sigma_{\eta}(x ; \bar{v})<0 .
$$

Indeed, arguing by contradiction, assume that any $\bar{v} \in \mathcal{D}$ satisfies

$$
\Sigma_{\eta}(x ; \bar{v}) \geq 0 .
$$

Let $\bar{v}_{0} \in \mathcal{D}$, then by (1.8) and (5.2), there exists $\bar{v}_{1} \in F(t, x)$ such that

$$
\Sigma_{\eta}\left(x ; \bar{v}_{1}-\bar{v}_{0}\right) \leq-\rho .
$$

Notice that

$$
\Sigma_{\eta}\left(x ; \bar{v}_{1}-v\right) \leq \Sigma_{\eta}\left(x ; \bar{v}_{1}-\bar{v}_{0}\right)+\Sigma_{\eta}\left(x ; \bar{v}_{0}-v\right) \leq-2 \rho,
$$

implying $\bar{v}_{1} \in \mathcal{D}$. Iterating the procedure we obtain a sequence $\left\{\bar{v}_{n}\right\}_{n \in \mathbb{N}} \subset \mathcal{D}$ satisfying, for any $n \in \mathbb{N}$,

$$
\Sigma_{\eta}\left(x ; \bar{v}_{n}-\bar{v}_{n-1}\right) \leq-\rho .
$$

Hence, for any $n \in \mathbb{N}$,

$$
\Sigma_{\eta}\left(x ; \bar{v}_{n}-\bar{v}_{0}\right) \leq \sum_{i=1}^{n} \Sigma_{\eta}\left(x ; \bar{v}_{i}-\bar{v}_{i-1}\right) \leq-n \rho,
$$

leading to a contradiction since by (2.3) and (2.8)

$$
\left|\Sigma_{\eta}\left(x ; \bar{v}-\bar{v}_{0}\right)\right| \leq M_{S}\left|\bar{v}-\bar{v}_{0}\right| \leq M_{S} M
$$

for any $\bar{v} \in F(t, x)$. The claim of Step 1 follows.

Step 2. We prove that there exists $\overline{\bar{v}} \in \mathcal{D}$ such that

$$
\Sigma_{\eta}(x ; \overline{\bar{v}}) \leq-\rho .
$$

Let $\bar{v} \in \mathcal{D}$ satisfy (5.1) and consider the continuous function in $[0,1]$

$$
\varphi(\lambda)=\Sigma_{\eta}(x ; \lambda \bar{v}+(1-\lambda) v) .
$$

Since $\varphi(0) \geq 0$ and $\varphi(1)<0$, we get $\varphi(\bar{\lambda})=0$ for some $\bar{\lambda} \in[0,1)$. Further, as $v, \bar{v} \in F(t, x)$ and $F$ has convex images, $\lambda \bar{v}+(1-\lambda) v \in F(t, x)$, for any $\lambda \in[0,1]$. Then, by (1.8), for some $\overline{\bar{v}} \in F(t, x)$,

$$
\Sigma_{\eta}(x ; \overline{\bar{v}}-\bar{\lambda} \bar{v}-(1-\bar{\lambda}) v) \leq-\rho,
$$

implying

$$
\Sigma_{\eta}(x ; \overline{\bar{v}}-v) \leq \Sigma_{\eta}(x ; \overline{\bar{v}}-\bar{\lambda} \bar{v}-(1-\bar{\lambda}) v)+\Sigma_{\eta}(x ; \bar{\lambda}(\bar{v}-v)) \leq-(1+\bar{\lambda}) \rho .
$$

Hence $\overline{\bar{v}} \in \mathcal{D}$. Further,

$$
\Sigma_{\eta}(x ; \overline{\bar{v}}) \leq \Sigma_{\eta}(x ; \overline{\bar{v}}-\bar{\lambda} \bar{v}-(1-\bar{\lambda}) v)+\varphi(\bar{\lambda}) \leq-\rho,
$$

yielding (5.3) and ending the proof.

In case some compactness is assumed, we can use (1.5) as the inward pointing condition. 
Proposition 5.2. Let $F: I \times X \rightsquigarrow X$ be a set-valued map satisfying (2.7)-(2.8) with $k_{R}, \phi \in L^{\infty}\left(I, \mathbb{R}^{+}\right)$. If

$$
F(\cdot, \bar{x}) \text { is continuous for any } \bar{x} \in \partial K \text {, }
$$

and

$$
F(t, \bar{x}) \text { is compact, for any } t \in I \text { and any } \bar{x} \in \partial K
$$

then, assumption (1.5) implies (4.5) (for a possibly smaller $\rho$ ).

Proof. Let $D$ be a compact set and $R>0$ be such that $D \subset R B$. Set $\alpha=\|\phi\|_{L^{\infty}\left(I ; \mathbb{R}^{+}\right)}$, $\beta=\left\|k_{2 R}\right\|_{L^{\infty}\left(I ; \mathbb{R}^{+}\right)}$and let $J \subset I$ be such that $\mu(J)=1$ and

$$
\begin{gathered}
F(t, x) \subset \alpha\left(1+\|x\|_{X}\right) B \quad \forall(t, x) \in J \times X, \\
F(t, x) \subset F(t, y)+\beta\|x-y\|_{X} B \quad \forall(t, x, y) \in J \times R B \times R B .
\end{gathered}
$$

By (5.6), for any $t \in J$ and any $x \in D$,

$$
F(t, x) \subset \alpha(1+R) B
$$

implying, in particular, that

$$
\|v-w\|_{X} \leq M:=2 \alpha(1+R),
$$

for any $v, w \in F(t, x)$. Observe also that, by (5.4), the inclusion (5.8) is valid for every $(t, x) \in I \times(\partial K \cap D)$.

Fix $\bar{x} \in \partial K \cap D$ and let $\rho>0$ be as in (1.5). As in the first step in the proof of Lemma 5.1 , for any $t \in I$ and $v \in F(t, \bar{x})$ satisfying $\sigma(\bar{x} ; v) \geq 0$, define

$$
\mathcal{D}_{t, v}=\{\bar{v} \in F(t, \bar{x}): \sigma(\bar{x} ; \bar{v}-v) \leq-\rho\} .
$$

By (1.5) we know that $\mathcal{D}_{t, v} \neq \emptyset$. The proof proceeds in three steps.

Step 1. We claim that there exists $\bar{v} \in \mathcal{D}_{t, v}$ satisfying

$$
\sigma(\bar{x} ; \bar{v})<0 .
$$

Arguing by contradiction, we assume that $\sigma(\bar{x} ; \bar{v}) \geq 0$, for any $\bar{v} \in \mathcal{D}_{t, v}$ and fix $\bar{v}_{0} \in \mathcal{D}_{t, v}$. Then by (1.5), there exists $\bar{v}_{1}$ such that

$$
\sigma\left(\bar{x} ; \bar{v}_{1}-\bar{v}_{0}\right) \leq-\rho .
$$

Further, $\bar{v}_{1} \in \mathcal{D}_{t, v}$, indeed

$$
\sigma\left(\bar{x} ; \bar{v}_{1}-v\right) \leq \sigma\left(\bar{x} ; \bar{v}_{1}-\bar{v}_{0}\right)+\sigma\left(\bar{x} ; \bar{v}_{0}-v\right) \leq-2 \rho .
$$

Iterating the procedure we obtain a sequence $\left\{\bar{v}_{n}\right\}_{n \in \mathbb{N}} \subset \mathcal{D}_{t, v}$ satisfying, for any $n \in \mathbb{N}$,

$$
\sigma\left(\bar{x} ; \bar{v}_{n}-\bar{v}_{n-1}\right) \leq-\rho \quad \text { hence } \quad \sigma\left(\bar{x} ; \bar{v}_{n}-\bar{v}_{0}\right) \leq \sum_{i=1}^{n} \sigma\left(\bar{x} ; \bar{v}_{n}-\bar{v}_{n-1}\right) \leq-n \rho .
$$

A contradiction, since by (2.3) and (2.8), for any $\bar{v} \in \mathcal{D}_{t, v}$,

$$
\left|\sigma\left(\bar{x} ; \bar{v}_{n}-\bar{v}_{0}\right)\right| \leq M
$$


implying the first claim.

Step 2. Now, we prove that

$$
\begin{gathered}
\exists \tilde{\rho}>0 \text { such that if } \sigma(\bar{x} ; v) \geq 0 \text { for some } t \in I, v \in F(t, \bar{x}), \\
\text { then } \exists \bar{v} \in F(t, \bar{x}) \text { satisfying } \max \{\sigma(\bar{x} ; \bar{v}-v), \sigma(\bar{x} ; \bar{v})\} \leq-\tilde{\rho} .
\end{gathered}
$$

Notice first that the set

$$
\mathcal{E}=\{(t, v) \in I \times X: v \in F(t, \bar{x}) \text { and } \sigma(\bar{x} ; v) \geq 0\}
$$

is compact. Indeed, let $\left(t_{n}, v_{n}\right) \in \mathcal{E}$, then up to a subsequence $t_{n} \rightarrow t \in I$. By the continuity assumptions on $F$, there exists $\bar{v}_{n} \in F(t, \bar{x})$ such that

$$
\left\|v_{n}-\bar{v}_{n}\right\|_{X} \leq \varepsilon_{n}, \quad \text { with } \varepsilon_{n} \rightarrow 0 .
$$

The compactness assumption (5.5) allows to deduce that, up to a subsequence, $\bar{v}_{n} \rightarrow \bar{v} \in$ $F(t, \bar{x})$ in $X$, implying that also $v_{n} \rightarrow \bar{v}$ in $X$. Since $\sigma\left(\bar{x} ; v_{n}\right) \geq 0$ for any $n \in \mathbb{N}$, also $\sigma(\bar{x} ; \bar{v}) \geq 0$, yielding that $(t, \bar{v}) \in \mathcal{E}$. Now, define for every $(t, v) \in \mathcal{E}$, the set

$$
\widehat{\mathcal{D}}_{t, v}=\left\{\bar{v} \in F(t, \bar{x}): \sigma(\bar{x} ; \bar{v}-v) \leq-\frac{\rho}{2}\right\} .
$$

As in the previous step we show that

$$
\inf _{\bar{v} \in \widehat{\mathcal{D}}_{t, v}} \sigma(\bar{x} ; \bar{v})=-\rho_{t, v}<0
$$

for some $\rho_{t, v}>0$. In order to prove the claim of Step 2, it remains to show that

$$
\inf \left\{\rho_{t, v}:(t, v) \in \mathcal{E}\right\}=\bar{\rho}>0 .
$$

Assume for a moment that $\bar{\rho}=0$. Then we can find a sequence $\left(t_{n}, v_{n}\right) \in \mathcal{E}$ such that, for any $\bar{v}_{n} \in \widehat{\mathcal{D}}_{t_{n}, v_{n}}$, there exist $\xi_{n} \in \partial d_{K}(\bar{x})$ satisfying

$$
\left\langle\xi_{n}, \bar{v}_{n}\right\rangle \geq-\frac{1}{n}
$$

Since $\mathcal{E}$ is compact, up to a subsequence $\left(t_{n}, v_{n}\right) \rightarrow(t, v) \in \mathcal{E}$. By Step 1 there exists $\bar{v} \in F(t, \bar{x})$ such that

$$
\langle\xi, \bar{v}-v\rangle \leq-\rho,\langle\xi, \bar{v}\rangle<0, \quad \text { for every } \xi \in \partial d_{K}(\bar{x}) .
$$

By continuity, there exist $\bar{v}_{n} \in F\left(t_{n}, \bar{x}\right)$ converging to $\bar{v}$ when $n \rightarrow \infty$. Then for all large $n$,

$$
\sup _{\xi \in \partial d_{K}(\bar{x})}\left\langle\xi, \bar{v}_{n}-v_{n}\right\rangle \leq-\frac{\rho}{2} .
$$

Therefore $\bar{v}_{n} \in \widehat{\mathcal{D}}_{t_{n}, v_{n}}$. Let $\xi_{n}$ be as above and consider its subsequence converging weaklystar to some $\bar{\xi} \in \partial d_{K}(\bar{x})$. Thus $\langle\bar{\xi}, \bar{v}\rangle \geq 0$ leading to a contradiction with (5.11).

Hence, taking $\tilde{\rho}=\min \{\rho / 2, \bar{\rho}\}$, we obtain the second claim.

Step 3. We will show that there exists $\eta, \tilde{\rho}>0$ such that, for any $t \in J, x \in D \cap(\partial K+\eta B)$, and $v \in F(t, x)$ satisfying

$$
\sup _{\tau \in[0, \eta]} \sigma(z ; S(\tau) v) \geq 0, \quad \text { for some } z \in B(x, \eta),
$$


we have

$$
\left\{\bar{v} \in F(t, x): \sup _{\tau \in[0, \eta], z \in B(x, \eta)}\{\sigma(z ; S(\tau)(\bar{v}-v)), \sigma(z ; S(\tau) \bar{v})\} \leq-\tilde{\rho}\right\} \neq \emptyset .
$$

Assume by contradiction that we can find sequences $\delta_{i} \rightarrow 0+$,

$t_{i} \in J, \quad x_{i} \in D \cap\left(\partial K+\frac{1}{i} B\right), \quad z_{i} \in B\left(x_{i}, \frac{1}{i}\right), \quad \xi_{i} \in \partial d_{K}\left(z_{i}\right), \quad 0 \leq \tau_{i} \leq \frac{1}{i}, \quad v_{i} \in F\left(t_{i}, x_{i}\right)$ such that

$$
\left\langle\xi_{i}, S\left(\tau_{i}\right) v_{i}\right\rangle \geq-\frac{1}{i}
$$

and, for any $\bar{v}_{i} \in F\left(t_{i}, x_{i}\right)$ there exist

$$
0 \leq s_{i}^{1}, s_{i}^{2} \leq \frac{1}{i}, \quad y_{i}^{1} \in B\left(x_{i}, \frac{1}{i}\right), \quad y_{i}^{2} \in B\left(x_{i}, \frac{1}{i}\right), \quad \zeta_{i}^{1}, \in \partial d_{K}\left(y_{i}^{1}\right), \quad \zeta_{i}^{2}, \in \partial d_{K}\left(y_{i}^{2}\right)
$$

satisfying

$$
\max \left\{\left\langle\zeta_{i}^{1}, S\left(s_{i}^{1}\right)\left(\bar{v}_{i}-v_{i}\right)\right\rangle,\left\langle\zeta_{i}^{2}, S\left(s_{i}^{2}\right) \bar{v}_{i}\right\rangle\right\}>-\delta_{i} .
$$

Passing to the limit $i \rightarrow \infty$ we obtain that, up to subsequences,

$t_{i} \rightarrow t \in I, x_{i}, z_{i} \rightarrow \bar{x} \in \partial K \cap D$ in $X, \xi_{i} \stackrel{*}{\rightarrow} \xi_{0}$ weakly-star in $X^{*}, v_{i} \rightarrow v \in F(t, \bar{x})$ in $X$.

Concerning the last limit, notice that by (5.4) and (5.7), for any $k \in \mathbb{N}$ there exists $i_{k}$ such that

$$
v_{i} \in F\left(t_{i}, x_{i}\right) \subset F(t, \bar{x})+\frac{1}{k} B, \quad \text { for any } i \geq i_{k}
$$

implying that $\left\|v_{i}-w_{i}\right\|_{X} \leq \frac{1}{k}$, for some $w_{i} \in F(t, \bar{x})$ and, as $F(t, \bar{x})$ is a compact set, up to a subsequence, $w_{i} \rightarrow v \in F(t, \bar{x})$ yielding $v_{i} \rightarrow v$. Further, by [12, Proposition 2.1.5], $\xi_{0} \in \partial d_{K}(\bar{x})$. Finally, as $\tau_{i} \rightarrow 0$, from (5.12) we deduce that

$$
0 \leq \lim _{i \rightarrow \infty}\left\langle\xi_{i}, S\left(\tau_{i}\right) v_{i}\right\rangle=\left\langle\xi_{0}, v\right\rangle .
$$

Then, from (5.10) there exists $\bar{v} \in F(t, \bar{x})$ such that

$$
\max \{\sigma(\bar{x} ; \bar{v}-v), \sigma(\bar{x} ; \bar{v})\} \leq-\tilde{\rho} .
$$

Let $\bar{v}_{i} \in F\left(t_{i}, x_{i}\right)$ such that $\bar{v}_{i} \rightarrow \bar{v}$ in $X$. Taking subsequences and keeping the same notation we obtain

$$
y_{i}^{1}, y_{i}^{2} \rightarrow \bar{x} \quad \text { in } X, \quad \zeta_{i}^{1} \stackrel{*}{\rightarrow} \zeta^{1}, \zeta_{i}^{2} \stackrel{*}{\rightarrow} \zeta^{2} \quad \text { weakly-star in } X^{*},
$$

and

$$
0 \leq \liminf _{i \rightarrow \infty} \max \left\{\left\langle\zeta_{i}^{1}, \bar{v}_{i}-v_{i}\right\rangle,\left\langle\zeta_{i}^{2}, \bar{v}_{i}\right\rangle\right\}=\max \left\{\left\langle\zeta^{1}, \bar{v}-v\right\rangle,\left\langle\zeta^{2}, \bar{v}\right\rangle\right\} .
$$

As $\zeta^{1}, \zeta^{2} \in \partial d_{K}(\bar{x}),(5.16)$ contradicts (5.14), ending the proof.

Proposition 5.3. Let $X$ be reflexive, $F: I \times X \rightsquigarrow X$ be a set-valued map with nonempty images, satisfying (5.4), (2.7)-(2.8) with $k_{R}, \phi \in L^{\infty}\left(I, \mathbb{R}^{+}\right)$, and that, for any $\bar{x} \in \partial K$, $F(t, \bar{x})$ is convex, and

$$
\text { the set-valued map } \partial d_{K}(\cdot) \text { is upper semicontinuous at } \bar{x} \text { and } \partial d_{K}(\bar{x}) \text { is compact. }
$$


Then, assumption (1.5) implies (4.5).

If $X$ is Hilbert, then (1.7) can be expressed in terms of normal vectors to $\partial K$ as in (4.13). The model in Example 3.3 provides a concrete situation in which this simplified version of the inward pointing condition can be used.

Proof of Proposition 5.3. The proof is similar to the one of Proposition 5.2, modifying suitably limits in (5.13) and (5.15), as outlined in the proof of Proposition 2 in [17].

Proposition 5.4. Assume $X$ is reflexive, (5.4) and that $k_{R}, \phi \in L^{\infty}\left(I, \mathbb{R}^{+}\right)$. If for any $\bar{x} \in \partial K, F(t, \bar{x})$ is convex, and the function $d_{K}$ is continuously differentiable on $\partial K$, then, assumption (1.6) implies (4.5).

Note that if $X$ is a Hilbert space and the state constraint $K$ is the ball $R B$ or the annulus $R B \backslash r B$, as in Sections 3.1 and 3.2, then $d_{K} \in C^{1}$ on a neighborhood of $\partial K$.

Proof of Proposition 5.4. Note that (1.6) implies immediately (1.5). Once (1.5) is proved, we verify (4.5), as in the proof of Proposition 5.3.

\section{LiPsChitz CONTINUITY OF THE VALUE FUNCTION}

The first application of our approximation theorems concerns the local Lipschitz continuity of the value function associated to the Mayer problem of optimal control theory. Namely, given a cost function $g: X \rightarrow \mathbb{R}$, and $x_{0} \in K$, consider the problem

$$
\min \left\{g(x(1)): x \in \mathcal{S}_{[0,1]}^{K}\left(x_{0}\right)\right\} .
$$

The corresponding value function $V: I \times X \rightarrow \mathbb{R} \cup\{+\infty\}$ is defined by

$$
V\left(t_{0}, y_{0}\right)=\inf \left\{g(x(1)): x \in \mathcal{S}_{\left[t_{0}, 1\right]}^{K}\left(y_{0}\right)\right\}, \quad \text { for any } t_{0} \in I, y_{0} \in X,
$$

with the convention that $\inf \emptyset=+\infty$.

Theorem 6.1. Assume (1.7), (2.5)-(2.8), and that $g$ is locally Lipschitz continuous on $K$, that is, for any $R>0$, there exists $g_{R}>0$ such that, for any $x, y \in R B \cap K$,

$$
|g(x)-g(y)| \leq g_{R}\|x-y\|_{X} .
$$

Then $V$ is continuous on $I \times K$ and the map $y_{0} \mapsto V\left(t_{0}, y_{0}\right)$ is locally Lipschitz continuous on $K$ uniformly in time, that is, for any $R>0$, there exist $l_{R}>0$ such that, for any $t_{0} \in I, y_{1}, y_{2} \in R B \cap K$,

$$
\left.\mid V\left(t_{0}, y_{1}\right)-V\left(t_{0}, y_{2}\right)\right) \mid \leq l_{R}\left\|y_{1}-y_{2}\right\|_{X} .
$$

Proof. The key ingredient is the neighboring feasible trajectory Theorem 4.1. Indeed, let $R>0$ be fixed, $c_{0}$ be as in Theorem 4.1 and take $y_{1} \neq y_{2} \in K \cap R B$. Let $\tilde{R}$ be such that any solution $x$ to (4.1) with the initial datum in $R B$ satisfies $x(t) \in \tilde{R} B$, for any $t$. By the definition of value function, we know that, for any $t_{0} \in I$, there exists $x_{1} \in \mathcal{S}_{\left[t_{0}, 1\right]}^{K}\left(y_{1}\right)$ such that

Taking the function

$$
g\left(x_{1}(1)\right) \leq V\left(t_{0}, y_{1}\right)+\left\|y_{1}-y_{2}\right\|_{X}
$$

$$
w_{2}(t)=S\left(t-t_{0}\right) y_{2}+\int_{t_{0}}^{t} S(t-s) f^{x_{1}}(s) \mathrm{d} s
$$


with $f^{x_{1}}$ the selection corresponding to $x_{1}$ in $(2.2)$, by $(2.3)$ we have, for any $t \in\left[t_{0}, 1\right]$,

$$
\left\|x_{1}(t)-w_{2}(t)\right\|_{X} \leq M_{S}\left\|y_{1}-y_{2}\right\|_{X} .
$$

Since for a.e. $s \in\left[t_{0}, 1\right]$,

$$
\begin{aligned}
f^{x_{1}}(s) \in F\left(s, x_{1}(s)\right) & \subset F\left(s, w_{2}(s)\right)+k_{\tilde{R}}(s)\left\|x_{1}(s)-w_{2}(s)\right\|_{X} \\
& \subset F\left(s, w_{2}(s)\right)+k_{\tilde{R}}(s) M_{S}\left\|y_{1}-y_{2}\right\|_{X},
\end{aligned}
$$

by an infinite dimensional version of the Filippov theorem, see [17, Lemma A.1], there exists $z_{2}$ solution to (4.1) such that $z_{2}\left(t_{0}\right)=y_{2}$ and for any $t \in\left[t_{0}, 1\right]$,

$$
\left\|w_{2}(t)-z_{2}(t)\right\|_{X} \leq M_{S} \mathrm{e}^{M_{S}\left\|k_{\tilde{R}}\right\|_{L^{1}}} \int_{t_{0}}^{t} k_{\tilde{R}}(s) M_{S}\left\|y_{1}-y_{2}\right\|_{X} d s \leq C_{1}\left\|y_{1}-y_{2}\right\|_{X}
$$

with $C_{1}=M_{S} \mathrm{e}^{M_{S}\left\|k_{\tilde{R}}\right\|_{L^{1}}}\left\|k_{\tilde{R}}\right\|_{L^{1}} M_{S}$. From Theorem 4.1, we obtain the existence of $x_{2} \in$ $\mathcal{S}_{\left[t_{0}, 1\right]}^{K}\left(y_{2}\right)$ such that

$\left\|x_{2}-z_{2}\right\|_{\mathcal{C}\left(\left[t_{0}, 1\right], X\right)} \leq c_{0}\left(\max _{t \in\left[t_{0}, 1\right]} \operatorname{dist}_{K}\left(z_{2}(t)\right)+\left\|y_{1}-y_{2}\right\|_{X}\right) \leq c_{0}\left(\left(C_{1}+M_{S}\right)+1\right)\left\|y_{1}-y_{2}\right\|_{X}$,

so that, for a positive constant $C_{2}$ depending only on $R$, and for any $t \in\left[t_{0}, 1\right]$,

$$
\left\|x_{2}(t)-x_{1}(t)\right\|_{X} \leq C_{2}\left\|y_{1}-y_{2}\right\|_{X}
$$

This estimate jointly with the definition of the value function imply that

$$
V\left(t_{0}, y_{2}\right) \leq g\left(x_{2}(1)\right) \leq g\left(x_{1}(1)\right)+g_{\tilde{R}} C_{2}\left\|y_{1}-y_{2}\right\|_{X} \leq V\left(t_{0}, y_{1}\right)+l_{R}\left\|y_{1}-y_{2}\right\|_{X},
$$

where $l_{R}=g_{\tilde{R}} C_{2}+1$. By interchanging $y_{1}$ and $y_{2}$ in the previous calculation, we obtain (6.1).

In order to prove the continuity of $V$ on $I \times K$, consider $t_{1}, t_{2} \in I$ and $y_{0} \in K$. Suppose $t_{1}<t_{2}$ and let $x \in \mathcal{S}_{\left[t_{1}, 1\right]}^{K}\left(y_{0}\right)$ such that

$$
g(x(1)) \leq V\left(t_{1}, y_{0}\right)+t_{2}-t_{1} .
$$

The properties of the value function ensure that

$$
V\left(t_{1}, y_{0}\right) \leq V\left(t_{2}, x\left(t_{2}\right)\right) \leq g(x(1)) \leq V\left(t_{1}, y_{0}\right)+t_{2}-t_{1} .
$$

Let $R>0$ be such that $x(t) \in R B$ for every $t \in\left[t_{1}, 1\right]$. Then we can conclude the proof by remarking that inequality (6.2), the Lipschitz regularity (6.1), and (2.8), imply

$$
\begin{aligned}
\left|V\left(t_{1}, y_{0}\right)-V\left(t_{2}, y_{0}\right)\right| & \leq V\left(t_{2}, x\left(t_{2}\right)\right)-V\left(t_{1}, y_{0}\right)+\left|V\left(t_{2}, x\left(t_{2}\right)\right)-V\left(t_{2}, y_{0}\right)\right| \\
& \leq t_{2}-t_{1}+l_{R}\left\|x\left(t_{2}\right)-y_{0}\right\|_{X} \\
& \leq t_{2}-t_{1}+l_{R}\left\|\left(S\left(t_{2}-t_{1}\right)-I d\right) y_{0}\right\|_{X}+l_{R}\left\|\int_{t_{1}}^{t_{2}} S\left(t_{2}-t\right) f^{x}(t) \mathrm{d} t\right\|_{X} \\
& \leq t_{2}-t_{1}+l_{R}\left\|\left(S\left(t_{2}-t_{1}\right)-I d\right) y_{0}\right\|_{X}+l_{R} M_{S}(1+R) \int_{t_{1}}^{t_{2}} \phi(t) \mathrm{d} t .
\end{aligned}
$$




\section{VARIATIONAL INCLUSIONS}

In this section, as application of the approximation Theorem 4.2, we prove a theorem on variational inclusions which is the infinite dimensional version of Theorem 4.1 in [18].

Theorem 7.1. Under assumptions of Theorem 4.1 or Theorem 4.2, let $x_{0} \in K, \bar{x} \in$ $\mathcal{S}_{\left[t_{0}, 1\right]}^{K}\left(x_{0}\right), h_{i} \rightarrow 0+, w_{i} \rightarrow w_{0} \in I_{K}\left(x_{0}\right)$ be such that $x_{0}+h_{i} w_{i} \in K$, for all $i$. Consider $a$ solution $w$ to the linearized inclusion

$$
\dot{w}(t) \in \mathbb{A} w(t)+d_{x} F\left(t, \bar{x}(t), f^{\bar{x}}(t)\right) w(t), \quad \text { for a.e. } t \in\left[t_{0}, 1\right]
$$

with $w\left(t_{0}\right)=w_{0}$ and subject to the constraint

$$
w(t) \in I_{K}(\bar{x}(t)), \quad \text { for a.e. } t \in\left[t_{0}, 1\right] .
$$

If

$$
\lim _{h \rightarrow 0+} \frac{1}{h} \max _{t \in\left[t_{0}, 1\right]} \operatorname{dist}_{K}(\bar{x}(t)+h w(t))=0,
$$

then for any $i$ there exists $x_{i} \in \mathcal{S}_{\left[t_{0}, 1\right]}^{K}\left(x_{0}+h_{i} w_{i}\right)$ such that

$$
\frac{x_{i}-\bar{x}}{h_{i}} \rightarrow w \quad \text { in } \mathcal{C}\left(\left[t_{0}, 1\right], X\right) \text {. }
$$

Proof. Fix a solution $w$ to (7.1) and let $\pi(s) \in d_{x} F\left(s, \bar{x}(s), f^{\bar{x}}(s)\right) w(s)$ a.e. be an integrable selection such that

$$
w(t)=S(t) w_{0}+\int_{0}^{t} S(t-s) \pi(s) d s \quad \forall t \in\left[t_{0}, 1\right] .
$$

By the definition of derivative, we obtain that

$$
\lim _{h \rightarrow 0+} \operatorname{dist}\left(\pi(t), \frac{F(t, \bar{x}(t)+h w(t))-f^{\bar{x}}(t)}{h}\right)=0, \quad \text { for a.e. } t \in\left[t_{0}, 1\right] .
$$

Set

$$
y_{i}(t)=\bar{x}(t)+h_{i}\left(w(t)+w_{i}-w_{0}\right) \quad \text { and } \quad \gamma_{i}(t)=\operatorname{dist}\left(f^{\bar{x}}(t)+h_{i} \pi(t), F\left(t, y_{i}(t)\right)\right) .
$$

From (2.7) and (7.3), we obtain that $\int_{t_{0}}^{1} \gamma_{i}(s) d s=o\left(h_{i}\right)$. Hence applying Lemma A.1 from [17] there exists $\tilde{x}_{i}$ solving (4.1) with initial datum $\tilde{x}_{i}\left(t_{0}\right)=x_{0}+h_{i} w_{i}$ such that

$$
\frac{\tilde{x}_{i}-y_{i}}{h_{i}} \rightarrow 0 \quad \text { in } \mathcal{C}\left(\left[t_{0}, 1\right], X\right), \quad \text { implying } \quad \frac{\tilde{x}_{i}-\bar{x}}{h_{i}} \rightarrow w \quad \text { in } \mathcal{C}\left(\left[t_{0}, 1\right], X\right) .
$$

Now consider the compact set $D_{0}=\cup_{i \in \mathbb{N}}\left\{x_{0}+h_{i} w_{i}\right\} \cup\left\{x_{0}\right\}$. In light of Theorem 4.2, there exists $c_{D_{0}}$ such that for any $i$, we can find $x_{i} \in \mathcal{S}_{\left[t_{0}, 1\right]}^{K}\left(x_{0}+h_{i} w_{i}\right)$ satisfying

$$
\left\|x_{i}-\tilde{x}_{i}\right\|_{\mathcal{C}\left(\left[t_{0}, 1\right], X\right)} \leq c_{D_{0}}\left(\max _{t \in\left[t_{0}, 1\right]} \operatorname{dist}_{K}\left(\tilde{x}_{i}(t)\right)+\frac{h_{i}}{i}\right) .
$$

Further,

$$
\begin{aligned}
\operatorname{dist}_{K}\left(\tilde{x}_{i}(t)\right) & \leq\left\|\tilde{x}_{i}(t)-y_{i}(t)\right\|_{X}+\operatorname{dist}_{K}\left(\bar{x}(t)+h_{i}\left(w(t)+w_{i}-w_{0}\right)\right) \\
& \leq\left\|\tilde{x}_{i}(t)-y_{i}(t)\right\|_{X}+h_{i}\left\|w_{i}-w_{0}\right\|_{X}+\operatorname{dist}_{K}\left(\bar{x}(t)+h_{i} w(t)\right) .
\end{aligned}
$$


Hence, assumption (7.2) allows to conclude. Indeed,

$$
\begin{aligned}
\| \frac{x_{i}-\bar{x}}{h_{i}} & -w\left\|_{\mathcal{C}\left(\left[t_{0}, 1\right], X\right)} \leq \frac{1}{h_{i}}\right\| \tilde{x}_{i}-x_{i}\left\|_{\mathcal{C}\left(\left[t_{0}, 1\right], X\right)}+\right\| \frac{\tilde{x}_{i}-\bar{x}}{h_{i}}-w \|_{\mathcal{C}\left(\left[t_{0}, 1\right], X\right)} \\
& \leq c_{D_{0}} \frac{1}{h_{i}}\left(\max _{t \in\left[t_{0}, 1\right]} \operatorname{dist}_{K}\left(\tilde{x}_{i}(t)\right)+\frac{h_{i}}{i}\right)+o(1) \\
& \leq \frac{c_{D_{0}}}{h_{i}}\left\|\tilde{x}_{i}-y_{i}\right\|_{\mathcal{C}\left(\left[t_{0}, 1\right], X\right)}+\frac{c_{D_{0}}}{h_{i}} \max _{t \in\left[t_{0}, 1\right]} \operatorname{dist}_{K}\left(\bar{x}(t)+h_{i} w(t)\right)+c_{D_{0}}\left\|w_{i}-w_{0}\right\|_{X}+o(1) \rightarrow 0,
\end{aligned}
$$

in light of (7.4), (7.5), ending the proof.

\section{Proofs of Theorems 4.1 And 4.2}

To prove the main theorems of Section 4, we need the following lemma.

Lemma 8.1. Under the assumptions of Theorem 4.1, for every $R_{0}>0$, there exist $c, \delta>0$ such that, for any $\varepsilon^{\prime}>0$, any $\bar{t} \in\left[t_{0}, 1\right]$, and any solution $y$ of (4.1) with $y\left(t_{0}\right) \in K \cap R_{0} B$ and $y(\bar{t}) \in K$, we can find a solution $x_{\varepsilon^{\prime}}$ of (4.1) satisfying

$$
\begin{gathered}
x_{\varepsilon^{\prime}}(\bar{t})=y(\bar{t}), \quad x_{\varepsilon^{\prime}}(t) \in \operatorname{Int} K, \text { for any } t \in(\bar{t},(\bar{t}+\delta) \wedge 1], \\
\text { and } \quad\left\|x_{\varepsilon^{\prime}}-y\right\|_{\mathcal{C}([\bar{t}, 1], X)} \leq c \cdot \max _{t \in[\bar{t}, 1]} \operatorname{dist}_{K}(y(t))+\varepsilon^{\prime} .
\end{gathered}
$$

The same conclusions are valid if $(2.5)-(2.8)$ hold true with $\phi \in L^{\infty}\left(I, \mathbb{R}^{+}\right), F$ is convex valued, and (1.7) is replaced by (1.8).

Proof. Fix $R_{0}>0$ and $\varepsilon^{\prime}>0$. Let $y$ be a solution of (4.1) satisfying the assumptions of the lemma. By the Gronwall lemma, in light of (2.2), (2.3), and (2.8), there exists $R>0$ depending only on $R_{0}$ such that

$$
y(t) \in \frac{R}{2} B \quad \text { and } \quad S(\tau) y(t) \in R B, \quad \text { for any } \tau \in I, t \in\left[t_{0}, 1\right] .
$$

We can assume, without loss of generality, that $\varepsilon^{\prime}<R$. Taking $\eta, \rho, M, J, k_{R}, \phi$ as in (1.7), (2.7), (2.8), we define

$$
C=M_{S} M\left(2 M_{S}\left\|k_{R}\right\|_{L^{1}} \mathrm{e}^{M_{S}\left\|k_{R}\right\|_{L^{1}}}+1\right), \quad c=\frac{2 C}{\rho},
$$

and choose

$$
0<\delta \leq \frac{1}{2 C} \min \{R, \eta\}
$$

such that, for any measurable $E \subset I$ with $\mu(E) \leq \delta$,

$$
\int_{E} k_{R}(s) \mathrm{d} s<\frac{\rho\left\|k_{R}\right\|_{L^{1}}}{\rho+2 C} \quad \text { and } \quad \int_{E} \phi(s) \mathrm{d} s<\frac{\eta}{2 M_{S}(1+R)} .
$$

Finally, we set

$$
\begin{array}{r}
\Gamma=\left\{s \in[\bar{t}, 1] \cap J: \text { for some } \tau_{0} \leq \eta, S\left(\tau_{0}\right) y(s) \in \partial K+\eta B\right. \text { and } \\
\left.\sup _{z \in B\left(S\left(\tau_{0}\right) y(s), \eta\right)} \sigma\left(z ; S\left(\tau_{0}\right) f^{y}(s)\right) \geq 0\right\}
\end{array}
$$


and

$(8.5)$

$$
T=\left\{\begin{array}{lr}
(\bar{t}+\delta) \wedge 1, \quad \text { if } \mu(\Gamma \cap[\bar{t},(\bar{t}+\delta) \wedge 1])<\frac{1}{C}\left[c \cdot \max _{t \in[\bar{t}, 1]} \operatorname{dist}_{K}(y(t))+\varepsilon^{\prime}\right] \\
\min \left\{s \geq \bar{t}: \mu(\Gamma \cap[\bar{t}, s])=\frac{1}{C}\left[c \cdot \max _{t \in[\bar{t}, 1]} \operatorname{dist}_{K}(y(t))+\varepsilon^{\prime}\right]\right\}, & \text { otherwise. }
\end{array}\right.
$$

By assumption (1.7) and the measurable selection theorem, there exists a measurable selection $\bar{v}(s) \in F(s, y(s))$ such that, for any $s \in \Gamma \cap[\bar{t}, T]$

$$
\left\|\bar{v}(s)-f^{y}(s)\right\|_{X} \leq M
$$

and

$$
\sup _{(\tau, z) \in \mathcal{A}^{\eta}(y(s))}\left\{\sigma\left(z ; S(\tau)\left[\bar{v}(s)-f^{y}(s)\right]\right), \sigma(z ; S(\tau) \bar{v}(s))\right\} \leq-\rho
$$

Define

$$
f_{\varepsilon^{\prime}}(s)= \begin{cases}\bar{v}(s), & \text { for every } s \in \Gamma \cap[\bar{t}, T] \\ f^{y}(s), & \text { otherwise. }\end{cases}
$$

Consider the trajectory

$$
\begin{aligned}
y_{\varepsilon^{\prime}}(t) & =S(t-\bar{t}) y(\bar{t})+\int_{\bar{t}}^{t} S(t-s) f_{\bar{\varepsilon}^{\prime}}(s) \mathrm{d} s \\
& =S(t-\bar{t}) y(\bar{t})+\int_{\Gamma \cap[\bar{t}, t \wedge T]} S(t-s) \bar{v}(s) \mathrm{d} s+\int_{[\bar{t}, t] \backslash(\Gamma \cap \bar{t}, t \wedge T])} S(t-s) f^{y}(s) \mathrm{d} s .
\end{aligned}
$$

Applying (2.2), (2.3), (8.2), (8.5), (8.6) and (8.8), we obtain that on $[\bar{t}, 1]$

$$
\begin{aligned}
\left\|y_{\varepsilon^{\prime}}(t)-y(t)\right\|_{X} & \leq \int_{\bar{t}}^{t}\left\|S(t-s)\left[f_{\varepsilon^{\prime}}(s)-f^{y}(s)\right]\right\|_{X} \mathrm{~d} s \leq M_{S} \int_{\Gamma \cap[\bar{t}, t \wedge T]}\left\|\bar{v}(s)-f^{y}(s)\right\|_{X} \mathrm{~d} s \\
& \leq M_{S} M \mu(\Gamma \cap[\bar{t}, t \wedge T]) \leq c \cdot \max _{t \in[\bar{t}, 1]} \operatorname{dist}_{K}(y(t))+\varepsilon^{\prime} .
\end{aligned}
$$

Hence, by (8.2), (8.3)

$$
\left\|y_{\varepsilon^{\prime}}(t)-y(t)\right\|_{X} \leq M_{S} M \delta \leq \frac{R}{2} .
$$

We can apply an infinite dimensional version of the Filippov Theorem, see [17, Lemma A.1], to deduce that there exists a solution $x_{\varepsilon^{\prime}}$ of $(4.1)$ with $x_{\varepsilon^{\prime}}(\bar{t})=y(\bar{t})$ satisfying for any $t \in[\bar{t}, 1]$

$$
\begin{gathered}
\left\|x_{\varepsilon^{\prime}}(t)-y_{\varepsilon^{\prime}}(t)\right\|_{X} \leq 2 M_{S} \mathrm{e}^{M_{S}\left\|k_{R}\right\|_{L^{1}}} M_{S} M \mu(\Gamma \cap[\bar{t}, t \wedge T]) \int_{\bar{t}}^{t} k_{R}(s) \mathrm{d} s, \\
\left\|f^{x_{\varepsilon^{\prime}}}(t)-f_{\bar{\varepsilon}^{\prime}}(t)\right\|_{X} \leq k_{R}(t) \mu(\Gamma \cap[\bar{t}, t \wedge T])\left(2 M_{S} \mathrm{e}^{M_{S}\left\|k_{R}\right\|_{L^{1}}} M_{S} M \int_{\bar{t}}^{t} k_{R}(s) \mathrm{d} s+2 M_{S} M\right) .
\end{gathered}
$$


Therefore

$$
\begin{aligned}
\left\|x_{\varepsilon^{\prime}}(t)-y(t)\right\|_{X} & \leq\left\|x_{\varepsilon^{\prime}}(t)-y_{\varepsilon^{\prime}}(t)\right\|_{X}+\left\|y_{\varepsilon^{\prime}}(t)-y(t)\right\|_{X} \\
& \leq 2 M_{S}\left\|k_{R}\right\|_{L^{1}} \mathrm{e}^{M_{S}\left\|k_{R}\right\|_{L^{1}}} M_{S} M \mu(\Gamma \cap[\bar{t}, t \wedge T])+M_{S} M \mu(\Gamma \cap[\bar{t}, t \wedge T]) \\
& =C \mu(\Gamma \cap[\bar{t}, t \wedge T]) \leq c \cdot \max _{t \in[\bar{t}, 1]} d_{K}(y(t))+\varepsilon^{\prime} .
\end{aligned}
$$

Further, by the definition of $\delta$ in $(8.3)$, for any $t \in[\bar{t},(\bar{t}+\delta) \wedge 1]$, we obtain

$$
\begin{gathered}
\left\|x_{\varepsilon^{\prime}}(t)-y(t)\right\|_{X} \leq \min \left\{\frac{R}{2}, \frac{\eta}{2}\right\} \\
\left\|x_{\varepsilon^{\prime}}(t)-y_{\varepsilon^{\prime}}(t)\right\|_{X} \leq \frac{C}{\left\|k_{R}\right\|_{L^{1}}} \mu(\Gamma \cap[\bar{t}, t \wedge T]) \int_{\bar{t}}^{t} k_{R}(s) \mathrm{d} s \leq \frac{\rho}{2} \mu(\Gamma \cap[\bar{t}, t \wedge T])
\end{gathered}
$$

and

$$
\left\|f^{x_{\varepsilon^{\prime}}}(t)-f_{\varepsilon^{\prime}}(t)\right\|_{X} \leq k_{R}(t) \mu(\Gamma \cap[\bar{t}, t \wedge T])\left(\frac{\rho}{2}+2 M_{S} M\right) .
$$

We claim that $x_{\varepsilon^{\prime}}(t) \in \operatorname{Int} K$ for any $t \in(\bar{t},(\bar{t}+\delta) \wedge 1]$. Assume first that $t \in(\bar{t},(\bar{t}+\delta) \wedge 1]$ satisfies $\mu(\Gamma \cap[\bar{t}, t \wedge T])=0$. This implies that $t \leq T$, since if $t>T, \mu(\Gamma \cap[\bar{t}, t \wedge T])=$ $\mu(\Gamma \cap[\bar{t}, T])=0$ yielding by the definition of $T$, the contradictory $t>(\bar{t}+\delta) \wedge 1=T$. Hence, we obtain that $\mu(\Gamma \cap[\bar{t}, t])=0$ and $x_{\varepsilon^{\prime}} \equiv y$ in $[\bar{t}, t]$, so our claim becomes $y(t) \in \operatorname{Int} K$. From the definition of $\Gamma$, we have that for a.e. $s \in[\bar{t}, t]$ and every $\tau \in[0, \eta]$ either $S(\tau) y(s) \notin \partial K+\eta B$ or $S(\tau) y(s) \in \partial K+\eta B$ and

$$
\sup _{\substack{\xi \in \partial d_{K}(z) \\ z \in B(S(\tau) y(s), \eta)}}\left\langle\xi, S(\tau) f^{y}(s)\right\rangle<0 .
$$

Observe that, by (2.8) and (8.4), for every $s \in[\bar{t}, t]$ we have

$$
\int_{\bar{t}}^{s}\left\|S(t-r) f^{y}(r)\right\|_{X} \mathrm{~d} r \leq \frac{\eta}{2}
$$

implying together with (2.5),

$$
d_{K}(S(t-s) y(s)) \leq d_{K}(S(t-\bar{t}) y(\bar{t}))+\left\|\int_{\bar{t}}^{s} S(t-r) f^{y}(r) \mathrm{d} r\right\|_{X} \leq \frac{\eta}{2} .
$$

Consequently, if there exists $\bar{s} \in[\bar{t}, t]$ such that $S(t-\bar{s}) y(\bar{s}) \notin \partial K+\eta B$, we obtain by $(8.11)$

$$
d_{K}(y(t)) \leq d_{K}(S(t-\bar{s}) y(\bar{s}))+\left\|\int_{\bar{s}}^{t} S(t-r) f^{y}(r) \mathrm{d} r\right\|_{X} \leq-\eta+\frac{\eta}{2}<0,
$$

yielding $y(t) \in \operatorname{Int} K$. Otherwise, for every $s \in[\bar{t}, t], S(t-s) y(s) \in \partial K+\eta B$ and

$$
\left.\sup _{\substack{\xi \in \partial d_{K}(z) \\ z \in B(S(t-s) y(s), \eta)}}\left\langle\xi, S(t-s) f^{y}(s)\right)\right\rangle<0 \text { for a.e. } s \in[\bar{t}, t] .
$$


Applying the mean value theorem (see [12]) and (2.2), there exist $z \in[y(t), S(t-\bar{t}) y(\bar{t})]$ and $\xi \in \partial d_{K}(z)$ such that

$$
\begin{aligned}
d_{K}(y(t)) & =d_{K}(S(t-\bar{t}) y(\bar{t}))+\langle\xi, y(t)-S(t-\bar{t}) y(\bar{t})\rangle \\
& =d_{K}(S(t-\bar{t}) y(\bar{t}))+\int_{\bar{t}}^{t}\left\langle\xi, S(t-s) f^{y}(s)\right\rangle \mathrm{d} s .
\end{aligned}
$$

Since, for any $s \in[\bar{t}, t]$,

$$
\begin{aligned}
\|z-S(t-s) y(s)\|_{X} & \leq\|z-y(t)\|_{X}+\|y(t)-S(t-s) y(s)\|_{X} \\
& \leq\|S(t-\bar{t}) y(\bar{t})-y(t)\|_{X}+\|y(t)-S(t-s) y(s)\|_{X} \leq \eta,
\end{aligned}
$$

by $(2.5),(8.13)$ and $(8.14)$ we have that $d_{K}(y(t))<0$, implying the claim.

Now we consider the case $\mu(\Gamma \cap[\bar{t}, t \wedge T])>0$. Notice that, by $(8.1)$ and $(8.9), x_{\varepsilon^{\prime}}(t) \in$ $R B$, for any $t \in[\bar{t},(\bar{t}+\delta) \wedge 1]$. Now, if there exists $\bar{s} \in[\bar{t}, t]$ such that $d_{K}(S(t-\bar{s}) y(\bar{s}))<-\eta$, then using (2.2), (2.8), and (8.4), we obtain

$$
\begin{aligned}
d_{K}\left(x_{\varepsilon^{\prime}}(t)\right) & \leq d_{K}(S(t-\bar{s}) y(\bar{s}))+\left\|x_{\varepsilon^{\prime}}(t)-S(t-\bar{t}) y(\bar{t})\right\|_{X}+\|S(t-\bar{t}) y(\bar{t})-S(t-\bar{s}) y(\bar{s})\|_{X} \\
& <-\eta+2 M_{S}(1+R) \int_{\bar{t}}^{t} \phi(s) \mathrm{d} s<0 .
\end{aligned}
$$

Therefore, recalling also (8.1) and (8.12), we can restrict our attention to the case where

$$
S(t-s) y(s) \in \partial K+\eta B \quad \forall s \in[\bar{t}, t],
$$

that is, $(t-s, z) \in \mathcal{A}^{\eta}(y(s))$ for every $s \in[\bar{t}, t]$ and $z \in B(S(t-s) y(s), \eta)$. By (8.7), we deduce that for all $s \in[\bar{t}, t] \cap \Gamma$

$$
\sup _{\substack{\xi \in \partial d_{K}(z) \\ z \in B(S(t-s) y(s), \eta)}}\left\{\left\langle\xi, S(t-s)\left[\bar{v}(s)-f^{y}(s)\right]\right\rangle,\langle\xi, S(t-s) \bar{v}(s)\rangle\right\} \leq-\rho .
$$

On the other hand, for a.e. $s \in[\bar{t}, t] \backslash \Gamma$ we have

$$
\sup _{\substack{\xi \in \partial d_{K}(z) \\ z \in B(S(t-s) y(s), \eta)}}\left\langle\xi, S(t-s) f^{y}(s)\right\rangle<0 .
$$

If $t \in(T,(\bar{t}+\delta) \wedge 1]$, then

$$
\mu(\Gamma \cap[\bar{t}, T])=\frac{1}{C}\left[c \cdot \max _{t \in[\bar{t}, 1]} \operatorname{dist}_{K}(y(t))+\varepsilon^{\prime}\right] .
$$

From the mean value theorem, as above, for some $z \in\left[y(t), x_{\varepsilon^{\prime}}(t)\right]$ and $\xi \in \partial d_{K}(z)$, we have

$$
d_{K}\left(x_{\varepsilon^{\prime}}(t)\right)=d_{K}(y(t))+\left\langle\xi, x_{\varepsilon^{\prime}}(t)-y(t)\right\rangle .
$$

Further, since by (8.9) and (8.11), for any $s \in[\bar{t}, t]$,

$$
\|z-S(t-s) y(s)\|_{X} \leq\|z-y(t)\|_{X}+\left\|\int_{s}^{t} S(t-r) f^{y}(r) \mathrm{d} r\right\|_{X} \leq\left\|x_{\varepsilon^{\prime}}(t)-y(t)\right\|_{X}+\frac{\eta}{2} \leq \eta,
$$


then $z \in B(S(t-s) y(s), \eta)$. Hence, from (8.2), (8.8), (8.10), (8.15), (8.17), and (8.18), we obtain

$$
\begin{aligned}
d_{K}\left(x_{\varepsilon^{\prime}}(t)\right) & =d_{K}(y(t))+\left\langle\xi, x_{\varepsilon^{\prime}}(t)-y_{\varepsilon^{\prime}}(t)\right\rangle+\left\langle\xi, y_{\varepsilon^{\prime}}(t)-y(t)\right\rangle \\
& \leq d_{K}(y(t))+\frac{\rho}{2} \mu(\Gamma \cap[\bar{t}, T])+\int_{\Gamma \cap[\bar{t}, T]}\left\langle\xi, S(t-s)\left[\bar{v}(s)-f^{y}(s)\right]\right\rangle \mathrm{d} s \\
& \leq d_{K}(y(t))-\frac{\rho}{2} \mu(\Gamma \cap[\bar{t}, T])=d_{K}(y(t))-\frac{\rho}{2 C} c \cdot \max _{s \in[\bar{t}, 1]} d_{K}(y(s))-\frac{\rho}{2 C} \varepsilon^{\prime}<0 .
\end{aligned}
$$

Then, $x_{\varepsilon^{\prime}}(t) \in \operatorname{Int} K$. Finally, let $t \in(\bar{t}, T]$. By the mean value theorem there exist $z \in\left[S(t-\bar{t}) y(\bar{t}), y_{\varepsilon^{\prime}}(t)\right]$ and $\xi \in \partial d_{K}(z)$ such that

$$
d_{K}\left(y_{\varepsilon^{\prime}}(t)\right)=d_{K}(S(t-\bar{t}) y(\bar{t}))+\left\langle\xi, y_{\varepsilon^{\prime}}(t)-S(t-\bar{t}) y(\bar{t})\right\rangle .
$$

Applying again (2.2), (2.8), and (8.4), we have for every $s \in[\bar{t}, t]$

$$
\begin{aligned}
\|z-S(t-s) y(s)\|_{X} & \leq\|z-S(t-\bar{t}) y(\bar{t})\|_{X}+\|S(t-\bar{t}) y(\bar{t})-S(t-s) y(s)\|_{X} \\
& \leq\left\|y_{\varepsilon^{\prime}}(t)-S(t-\bar{t}) y(\bar{t})\right\|_{X}+\|S(t-\bar{t}) y(\bar{t})-S(t-s) y(s)\|_{X} \\
& \leq 2 M_{S}(1+R) \int_{\bar{t}}^{t} \phi(r) \mathrm{d} r \leq \eta .
\end{aligned}
$$

Then from (8.8), (8.10), (8.15), (8.16) and (8.19), we obtain

$$
\begin{aligned}
d_{K}\left(x_{\varepsilon^{\prime}}(t)\right) & \leq\left\|x_{\varepsilon^{\prime}}(t)-y_{\varepsilon^{\prime}}(t)\right\|_{X}+d_{K}\left(y_{\varepsilon^{\prime}}(t)\right) \\
& \left.\leq \frac{\rho}{2} \mu(\Gamma \cap[\bar{t}, t])+d_{K}(S(t-\bar{t}) y(\bar{t}))+\int_{\bar{t}}^{t}\left\langle\xi, S(t-s) f_{\varepsilon^{\prime}}(s)\right)\right\rangle \mathrm{d} s \\
& \leq \frac{\rho}{2} \mu(\Gamma \cap[\bar{t}, t])+\int_{\Gamma \cap[\bar{t}, t]}\langle\xi, S(t-s) \bar{v}(s)\rangle \mathrm{d} s+\int_{[\bar{t}, t] \backslash \Gamma}\left\langle\xi, S(t-s) f^{y}(s)\right\rangle \mathrm{d} s \\
& \leq\left(\frac{\rho}{2}-\rho\right) \mu(\Gamma \cap[\bar{t}, t])<0 .
\end{aligned}
$$

Also in this case $x_{\varepsilon^{\prime}}(t) \in \operatorname{Int} K$.

If (2.5)-(2.8) are valid with $\phi \in L^{\infty}\left(I, \mathbb{R}^{+}\right)$, convex valued $F$, and (1.7) replaced by (1.8), the same claim follows from Lemma 5.1 and the proof above.

Proof of Theorem 4.1. The construction of the trajectory $x$, based on Lemma 8.1, proceeds exactly as in [18, p.745].

Proof of Theorem 4.2. Notice that, by the uniform continuity of the semigroup $S(t)$ over compact sets, we have that for any compact $D$ and any $\eta>0$, there exists $0<\tilde{\eta} \leq \eta / 2$ such that, for any $\tau \leq \tilde{\eta}$ and any $x \in D,\|S(\tau) x-x\|_{X} \leq \eta / 2$. Then, it is possible to prove that assumption (4.5) implies

$$
\begin{aligned}
& \forall D \subset X \text { compact, } \exists \tilde{\eta}, \rho, M>0, \exists J \subset I \text { such that } \mu(J)=1 \text { and whenever } \\
& \qquad \Sigma_{\tilde{\eta}}(x ; v) \geq 0 \text { for some } t \in I, x \in D \cap \partial^{\tilde{\eta}} K, v \in F(t, x), \\
& \text { then } \exists \bar{v} \in F(t, x) \cap B(v, M) \text { satisfying } \max \left\{\Sigma_{\tilde{\eta}}(x ; \bar{v}-v) ; \Sigma_{\tilde{\eta}}(x ; \bar{v})\right\} \leq-\rho .
\end{aligned}
$$


Then, following the same proof as the one of Lemma 8.1 we obtain that for every compact $D_{0} \subset X$ there exist $c, \delta>0$ such that, for any $\varepsilon^{\prime}>0$, any $\bar{t} \in\left[t_{0}, 1\right]$, and any solution $y$ of (1.3) with $y\left(t_{0}\right) \in K \cap D_{0}$ and $y(\bar{t}) \in K$, we can find a solution $x_{\varepsilon^{\prime}}$ of (1.3) satisfying

$$
\begin{gathered}
x_{\varepsilon^{\prime}}(\bar{t})=y(\bar{t}), \quad x_{\varepsilon^{\prime}}(t) \in \operatorname{Int} K, \text { for any } t \in(\bar{t},(\bar{t}+\delta) \wedge 1], \\
\text { and }\left\|x_{\varepsilon^{\prime}}-y\right\|_{L^{\infty}([\bar{t}, 1], X)} \leq c \cdot \max _{t \in[\bar{t}, 1]} \operatorname{dist}_{K}(y(t))+\varepsilon^{\prime} .
\end{gathered}
$$

This allows to conclude as in Theorem 4.1.

With the help of Lemma 5.1, the same conclusions hold true if (2.5)-(2.8) are valid with $\phi \in L^{\infty}\left(I, \mathbb{R}^{+}\right), F$ is convex valued, and (1.7) is replaced by (1.8).

\section{REFERENCES}

[1] W. Arendt Semigroups and evolution equations: functional calculus, regularity and kernel estimates. Evolutionary equations. Vol. I, 1-85, Handb. Differ. Equ. North-Holland, Amsterdam, 2004.

[2] W. Arendt, G. Metafune, D. Pallara, S. Romanelli, The Laplacian with Wentzell-Robin boundary conditions on spaces of continuous functions, Semigroup Forum 67 (2003), 247-261.

[3] J.Y Beale, S.I. Rosencrans, Acoustic boundary conditions, Bull. Amer. Math. Soc. 80 (1974), 12761278.

[4] P. Bettiol, A. Bressan, R. B. Vinter, On trajectories satisfying a state constraint: $W^{1,1}$ estimates and counter-examples, SIAM J. Control Optim. 48 (2010), 4664-4679.

[5] P. Bettiol, H. Frankowska, R. B. Vinter, $L^{\infty}$ estimates on trajectories confined to a closed subset, J. Differential Equations 252 (2012), 1912-1933.

[6] H. Brézis, Analyse fonctionnelle: théorie et applications, Masson, Paris, 1983.

[7] H. Brezis, A. Pazy, Semigroups of nonlinear contractions on convex sets, J. Funct. Anal. 6 (1970), 237-281.

[8] P. Cannarsa, H. Frankowska, Value function and optimality conditions for semilinear control problems, Appl. Math. Optim. 26 (1992), 139-169.

[9] P. Cannarsa, H. Frankowska, E. M. Marchini, On Bolza optimal control problems with constraints, Discrete Contin. Dyn. Syst. 11 (2009), 629-653.

[10] P. Cannarsa, H. Frankowska, E.M. Marchini, Optimal control for evolution equations with memory, J. Evol. Equ. 13 (2013), 197-227.

[11] K.N. Chueh, C.C. Conley, J.A. Smoller, Positively invariant regions for systems of nonlinear equation, Indiana Univ. Math. J. 26 (1977), 373-392.

[12] F. H. Clarke, Optimization and nonsmooth analysis, SIAM, Philadelphia, 1990.

[13] G.M. Coclite, G.R. Goldstein, J.A. Goldstein, Stability estimates for nonlinear hyperbolic problems with nonlinear Wentzell boundary conditions, Z. Angew. Math. Phys. 64 (2013), 733-753.

[14] C. M. Dafermos: Asymptotic stability in viscoelasticity, Arch. Ration. Mech. Anal. 37 (1970), 297308.

[15] K.-J. Engel, The Laplacian on $\mathcal{C}(\bar{\Omega})$ with generalized Wentzell boundary conditions, Arch. Math. 81 (2003), 548-558.

[16] K.-J. Engel, R. Nagel, One-Parameter Semigroups for Linear Evolution Equations, Springer-Verlag, New York, 2000.

[17] H. Frankowska, E.M. Marchini, M. Mazzola, A relaxation result for state constrained inclusions in infinite dimension, Mathematical Control and Related Fields (MCRF) 6 (2016), 113-141.

[18] H. Frankowska, M. Mazzola, Discontinuous solutions of Hamilton-Jacobi-Bellman equation under state constraints, Calc. Var. Partial Differential Equations 46 (2013), 725-747.

[19] H. Frankowska, M. Mazzola, On relations of the adjoint state to the value function for optimal control problems with state constraints, Nonlinear Differ. Equ. Appl. 20 (2013), 361-383. 
[20] H. Frankowska, F. Rampazzo, Filippov's and Filippov-Wazewski's theorems on closed domains, J. Differential Equations 161 (2000), 449-478.

[21] H. Frankowska, R.B. Vinter, Existence of neighbouring feasible trajectories: Applications to dynamic programming for state constrained optimal control problems, J. Optim. Theory Appl. 104 (2000), $21-40$.

[22] C.G. Gal, G.R. Goldstein, J.A. Goldstein, Oscillatory boundary conditions for acoustic wave equations, J. Evol. Equ. 3 (2003), 623-635.

[23] G.R. Goldstein. Derivation and physical interpretation of general boundary conditions, Adv. Diff. Eqns. 11 (2006), 457-480.

[24] P.M. Moser, K.U. Ingard, Theoretical acoustics, McGraw Hill, New York, 1968.

[25] E.M. Ouhabaz, Invariance of closed convex sets and domination criteria for semigroups, Potential Analysis 5 (1996), 611-625.

[26] A. Pazy, Semigroups of linear operators and applications to partial differential equations, SpringerVerlag, New York, 1983.

[27] H. M. Soner, Optimal control with state-space constraints, SIAM J. Control Optim. 24 (1986), 552561.

[28] R. Temam, Infinite-dimensional dynamical systems in mechanics and physics, Springer, New York, 1997.

[29] J.L. Vazquez, E. Vitillaro, Heat equation with dynamical boundary conditions of locally reactive type, Semigroup Forum 74 (2007), 1-40.

CNRS, Institut de Mathématiques de Jussieu - Paris Rive Gauche, UMR 7586,

Sorbonne Universités, UPMC Univ Paris 06, Univ Paris Diderot, Sorbonne Paris Cité, Case 247, 4 Place Jussieu, 75252 Paris, France

E-mail address: helene.frankowska@imj-prg.fr

Dipartimento di Matematica "F.Brioschi", Politecnico di Milano

Piazza Leonardo da Vinci 32, 20133 Milano, Italy

E-mail address: elsa.marchini@polimi.it

Sorbonne Universités, UPMC Univ Paris 06, Institut de Mathématiques de Jussieu Paris Rive Gauche, UMr 7586, CNRS, Univ Paris Diderot, Sorbonne Paris Cité, Case 247, 4 Place Jussieu, 75252 Paris, France

E-mail address: marco.mazzola@imj-prg.fr 